\title{
Adaptación psicométrica de la Escala de Depresión para Adolescentes de Reynolds (EDAR) en estudiantes de secundaria de Lima metropolitana
}

\author{
Nelly Ugarriza \& Miguel Escurra* \\ Universidad de Lima \\ Lima, Perú
}

Se presentan normas percentilares de la Escala de Depresión para Adolescentes de Reynolds (EDAR). La muestra estuvo integrada por 1.963 estudiantes secundarios, varones y mujeres, de colegios estatales y privados de Lima metropolitana. Los coeficientes alfa obtenidos por encima de .87 demostraron la consistencia interna de la EDAR. Se halló que la depresión era mayor en las mujeres y en los escolares de colegios estatales. El procedimiento de análisis factorial Varimax dio lugar a una solución de seis factores rotados, que explicaba el 45,8\% de la varianza: desmoralización, cólera y tristeza, anhedonia, baja autoestima, somático-vegetativo y ansiedad escolar.

depresión / validez / factorial / confiabilidad / normas

\section{Psychometric adaptation of the Reynolds Adolescent Depression Scale in Peruvian secondary students in metropolitan Lima}

Percentile norms are presented for the Reynolds Adolescent Depression Scale (RADS). They were obtained from a sample of 1.963 Peruvian secondary students, all men and women, from public and private schools. The alpha coefficients obtained were above .87, showing the internal consistency of the scale. It was found that depression is higher in women and in public schools' students. Factorial analyses were performed: the rotated six factor orthogonal solution (Varimax) was obtained accounting for 45,8 percent of the total variance: Demoralization, anger and sadness, anhedonia, low self-esteem, somaticvegetative and school anxiety.

depression / factorial / validity / reliability / norms

* Agradecemos la colaboración de los alumnos Mónica Flores, Rosalie Harten, Miguel Ángel Linares, Paola Lindo, Jorge Luna y Ana Teresa Piccini, de la Facultad de Psicología de la Universidad de Lima. Dirección de los autores: nellyugar@terra.com.pe, mescurra@terra.com.pe 
La depresión, como problema de salud de la adolescencia, ha adquirido una creciente importancia a la luz del aumento en las tasas de suicidio en varios países. Asimismo, el incremento de otros problemas emocionales y conductuales, como los problemas de conducta, el consumo de alcohol y drogas, el embarazo precoz y el fracaso escolar, entre otros, pueden estar relacionados con una sintomatología depresiva. Por esta razón, es importante desarrollar estudios que permitan ampliar el marco teórico y metodológico de este trastorno.

Uno de los modelos teóricos que mayor influencia ha tenido en la explicación de la depresión es el modelo cognitivo de Beck, quien inició una serie de trabajos con la idea de validar la tesis psicoanalítica sobre el trastorno depresivo. Ésta se basa en la explicación de una historia temprana de desamparo, que desarrolla en la persona sentimientos de hostilidad hacia aquellas figuras significativas que lo abandonaron de manera real o simbólica. La hostilidad hacia estos objetos es reprimida -por ser inaceptable- volviéndose hacia sí misma, es decir, la depresión no sería más que hostilidad dirigida hacia uno mismo, expresada como "necesidad de sufrimiento".

Pero la investigación empírica de Beck no respaldaba la tesis de que los pacientes tuviesen necesidad de sufrir, por el contrario, los resultados señalaban que éstos más bien evitaban con- ductas que pudieran generar rechazo o desaprobación y sus verbalizaciones expresaban una visión negativa de sí mismos y de su vida.

Las personas deprimidas poseen "esquemas" distorsionados de la realidad, que fácilmente se activan ante sucesos estresantes (Beck, 1976). Estos esquemas, al orientar y canalizar la percepción de la realidad, generan una visión negativa de sí mismo, del mundo y del futuro, que produce emociones indeseables.

Las causas de la depresión son las ideas, pensamientos, y creencias específicas que tienen sobre el mundo y sobre sí mismos. Se asume que los síntomas depresivos son consecuencia de un estado emocional alterado y éste, a su vez, tiene su origen en una serie de "cogniciones peculiares", debiendo además existir un elemento estresante que active las cogniciones depresivas latentes, de lo contrario no se produce la depresión. Así, ni las cogniciones depresivas ni el estrés por sí solo pueden dar cuenta de una reacción depresiva.

Detrás de la depresión ocurre una serie de pensamientos disfuncionales o pensamientos negativos sobre sí mismo y del futuro, que Beck denominó la tríada cognitiva de la depresión. Además existen factores fisiológicos que desempeñan también una función muy importante en la etiología de las depresiones, además del rol que ejerce la conducta y el ambiente como iniciadores y reforzadores de ésta. Sin embar- 
go, el factor cognitivo - sea cual fuere la causa de una depresión- siempre está presente.

\section{DEPRESIÓN EN ADULTOS Y ADOLESCENTES}

La información proporcionada por una encuesta realizada por el Instituto Nacional de Salud Mental (Robins, Helzer, Weissman, Orvaschel, Gruenberg, Burke, \& Regier, 1984) indica que los desórdenes depresivos son experimentados por el 6 al 10\% de la población a lo largo de la vida. La depresión, más que un desorden del estado de ánimo, afecta todas las áreas del funcionamiento humano, incluyendo los campos comportamentales, somáticos, emocionales y cognitivos. Lo más importante es que los desórdenes depresivos constituyen siempre una amenaza potencial para la vida si no son identificados y tratados.

Las dos últimas décadas han sido testigos de un creciente interés, de parte de los clínicos e investigadores, en el examen de la depresión en adultos y adolescentes. A esto le siguió un amplio periodo durante el cual la depresión fue vista como no existente, oculta o expresada en sintomatologías bastante diferentes a las de los adultos. Esta última perspectiva fue expresada principalmente a través de constructos equivalentes a la depresión (Cytryn \& McKnew, 1972; Glaser, 1967; Hollon, 1970; Rie, 1966), en los cuales se presumió que la depresión se manifestaba mediante comportamientos como la agresión, hiperactividad y delincuencia. Actualmente se considera que la depresión se manifiesta en la adolescencia y en la adultez bajo los mismos síntomas, tal como lo señalan la Asociación Psiquiátrica Americana (1980) e investigadores como Carlson \& Strober, 1979; Christ, Adler, Isacoff \& Gershansky, 1981, Reynolds 1985a; Yanchyshyn \& Robbins, 1983).

En los adultos la depresión se expresa no como un síntoma aislado (por ejemplo, manifestar humor triste), pero sí como un conglomerado de síntomas que pueden incluir baja autoestima, anhedonia, aislamiento social, fatiga, bajo rendimiento académico, ortografía defectuosa, disturbios en la conducta alimenticia, en los trastornos del sueño e impulsos autodestructivos (American Psychiatric Association, 1980; Carlson \& Strober, 1979; Strober, Green, \& Carlson, 1981). En las entrevistas clínicas los adolescentes depresivos demostraron el mismo rango de síntomas asociados con la depresión en adultos (Friedman, Hurt, Clarkin, Corn, \& Aronoff, 1983).

Es importante no visualizar la sintomatología de la depresión como un aspecto normal del desarrollo adolescente, los síntomas depresivos no son equivalentes a los conceptos difusos de las alteraciones adolescentes. La depresión en los adolescentes constituye un desorden psicológico que si no es tratado puede persistir por meses o años, y en su 
forma más severa puede tener consecuencias amenazadoras para la vida.

El Manual Estadístico y de Diagnóstico de Desórdenes Mentales (DSMIV) considera la misma sintomatología en el denominado episodio depresivo mayor, al cual describe como un período de al menos dos semanas en el que se da un estado de ánimo deprimido o una pérdida de interés o placer en casi todas las actividades. En los niños y adolescentes este estado de ánimo puede ser irritable en lugar de triste. La persona también debe experimentar al menos cuatro síntomas de una lista que incluye cambios de apetito o peso, del sueño y de la actividad psicomotora; falta de energía; sentimientos de infravaloración o culpa; dificultad para pensar, concentrarse o tomar decisiones, y pensamientos recurrentes de muerte o ideación, planes o intentos suicidas.

Para indicar la existencia de un episodio depresivo mayor, un síntoma debe ser de nueva presentación o haber empeorado claramente si se compara con el estado del sujeto antes del episodio. Los síntomas han de mantenerse la mayor parte del día, casi cada día, durante al menos dos semanas consecutivas. El episodio debe acompañarse de un malestar clínico significativo o de deterioro social, laboral o de otras áreas importantes de la actividad del individuo. En algunos sujetos con episodios leves la actividad puede parecer normal, pero a costa de un esfuerzo muy importante.

Frecuentemente, el estado de ánimo en un episodio depresivo mayor es descrito por el sujeto como deprimido, triste, desesperanzado, desanimado. En algunos casos, la tristeza puede ser negada al principio, pero más tarde puede ser suscitada por la entrevista (por ejemplo, al señalar que el sujeto parece que está a punto de llorar). La presencia de un estado de ánimo depresivo puede inferirse de la expresión facial y el comportamiento aparente del sujeto. Algunas personas ponen énfasis en las quejas somáticas en lugar de referir sentimientos de tristeza. Muchos sujetos refieren o muestran una alta irritabilidad (por ejemplo, ira persistente, tendencia a responder a los acontecimientos con arranques de ira o insultando a los demás, o sentimiento exagerado de frustración por cosas sin importancia). En los niños y adolescentes, más que un estado de ánimo triste o desanimado, puede presentarse u estado de ánimo irritable o inestable. Esta forma de presentación debe diferenciarse del patrón de "niño mimado" con irritabilidad ante las frustraciones.

Casi siempre hay una pérdida de intereses y de capacidad para el placer en mayor o menor medida. Las personas pueden referir el sentimiento de estar menos interesados en sus aficiones, de que "ha perdido el interés" o de haber dejado de disfrutar con actividades que antes consideraban placenteras. Frecuentemente, son los familiares los que notan el aislamiento social o el abandono de aficiones. En algunos sujetos hay 
una reducción significativa de los niveles previos de interés o de deseo sexual.

Normalmente, el apetito disminuye y mucho sujetos tienen que esforzarse por comer. Otros, especialmente en la consulta ambulatoria, pueden tener más apetito, con apetencia por ciertas comidas. Cuando las alteraciones del apetito son graves (sea en la dirección que sea), puede haber una pérdida o ganancia significativa de peso o, en niños, se puede dar un fracaso en la consecución del peso apropiado.

La alteración del sueño, asociada más a menudo a un episodio depresivo mayor, es el insomnio. Es característico el insomnio medio (despertarse durante la noche y tener problemas para volver a dormirse) o el insomnio tardío (despertarse demasiado pronto y ser incapaz de volver a dormirse). También se puede presentar un insomnio inicial (problemas para conciliar el sueño). Menos frecuente, los sujetos se quejan de exceso de sueño (hipersomnia) en forma de episodios de sueño prolongado nocturno o de aumento del sueño diurno. Algunas veces el trastorno del sueño es la razón por la que el sujeto acude en busca de tratamiento.

Los cambios psicomotores incluyen agitación (la incapacidad para permanecer sentado, paseos, frotarse las manos, pellizcar o arrugar la piel, la ropa o algún objeto) o enlentecimiento (en el lenguaje, pensamiento y movimientos corporales lentos; aumento de la latencia de respuesta, bajo volumen de la voz, menos inflexiones y cantidad o variedad de contenido, o mutismo). La asignación o el enlentecimiento psicomotores deben ser lo bastante graves como para ser observables por los demás y no representar únicamente una sensación subjetiva.

Es habitual la falta de energía, el cansancio y la fatiga. Una persona puede referir una fatiga persistente sin hacer ejercicio físico. Incluso el menor trabajo parece requerir un gran esfuerzo. Puede reducirse la eficacia con que se realizan las tareas rutinarias.

El sentimiento de inutilidad o de culpa, asociada a un episodio mayor, puede implicar evaluaciones negativas no realistas del propio valor, preocupaciones o el culparse constantemente por pequeños errores pasados. Se suele malinterpretar los acontecimientos cotidianos neutros o triviales, tomándolos como pruebas de sus defectos personales, y tener un exagerado sentimiento de responsabilidad por las adversidades. El sentimiento de inutilidad o de culpa puede tener proporciones delirantes (un sujeto está convencido de que está personalmente implicado en la pobreza del mundo). Es muy frecuente el autorreproche de estar enfermo o de no haber logrado cumplir con las responsabilidades laborales o interpersonales como resultado de la depresión, pero a no ser que alcance una magnitud delirante, esta queja no se considera dentro de este criterio.

Muchas personas refieren una capacidad disminuida para pensar, concen- 
trarse o tomar decisiones. Pueden dar la impresión de distraerse con facilidad o quejarse de falta de memoria. En sujetos de edad avanzada con un episodio depresivo mayor, la queja principal puede ser la falta de memoria, que puede ser tomada erróneamente por un signo de demencia ("pseudodemencia"). Cuando es tratado con éxito el episodio depresivo mayor, suelen desaparecer los problemas de memoria. Sin embargo, en algunos sujetos, especialmente ancianos, el episodio depresivo mayor puede ser la presencia inicial de una demencia irreversible. En los niños, la caída repentina de los resultados en las notas puede ser un reflejo de la falta de concentración.

Son frecuentes los pensamientos de muerte, la ideación suicida o las tentativas suicidas. Estas ideas varían desde la creencia consistente en que los demás estarían mejor si uno muriese, hasta los pensamientos transitorios, pero recurrentes, sobre el hecho de suicidarse, o los auténticos planes específicos sobre cómo cometer el suicidio. La frecuencia, intensidad y letalidad de estas ideas pueden ser muy variables. Si bien estos comportamientos están asociados estadísticamente con las tentativas de suicidio y pueden ser útiles para la identificación del grupo de alto riesgo, muchos estudios han demostrado que no es posible predecir con exactitud cuándo o en qué momento un determinado sujeto deprimido va a intentar el suicidio. Los motivos para el suicidio pueden incluir el deseo de rendirse ante lo que es percibido como obstáculos insalvables o un intenso deseo de acabar con un estado emocional enormemente doloroso, que es percibido como interminable.

El diagnóstico de episodio depresivo mayor no se realiza si los síntomas cumplen los criterios para un episodio mixto. Éste se caracteriza por presentar síntomas tanto de un episodio maníaco como de un episodio depresivo mayor prácticamente a diario, durante al menos una semana.

\section{Sistema DE DIAGNÓSTICo Y \\ CLASIFICACIÓN DE LA DEPRESIÓN}

Las actuales clasificaciones de la depresión utilizan un enfoque taxonómico para diferenciar los subtipos de la depresión, basados en un conjunto de síntomas, empleando tanto criterios de inclusión como de exclusión. Actualmente, el principal sistema para la clasificación de desórdenes afectivos en los adolescentes, niños y adultos es el Manual Estadístico y de Diagnóstico de Desórdenes Mentales (DSM-III y IV), su amplia aceptación profesional de la taxonomía ha brindado un criterio formal común para el diagnóstico de la depresión en adolescentes. Otro sistema de clasificación es el Research Diagnostic Criteria (RDC) sugerido por Spitzer, Endicott y Robins (1978). Mientras que el RDC es semejante al criterio del DSM-III, sin embargo existen diferencias fundamentales. El RDC 
brinda una mayor especificación de los distintos subtipos de la depresión y ha sido diseñado principalmente para estudios de investigación. El RDC es evaluado por una entrevista de diagnóstico clínico formal del Programa para Desórdenes Afectivos y Esquizofrenia (SADS) (Endicott \& Spitzer, 1978). También es usado pero con menor frecuencia el Criterio Weinberg (Weinberg, Rutman, Sullivan, Penick, \& Dietz, 1973), primer sistema formal de clasificación de los desórdenes depresivos específico en niños y adolescentes.

De acuerdo con las definiciones del DSM-III, los desórdenes depresivos se ubican en la categoría de desórdenes afectivos. Estos desórdenes incluyen alta depresión, desórdenes bipolares, ciclotímicos y distímicos. Los desórdenes son diferenciados por un conglomerado de síntomas, edad de inicio y duración del síntoma. Un punto importante para el estudio de la depresión en los adolescentes es la distinción entre los desórdenes distímicos y la depresión alta. El primero es el menos severo en términos de síntomas de distrés, pero típicamente es de mayor duración que la alta depresión y puede ser especialmente de mayor relevancia para el estudio de la depresión en las poblaciones de adolescentes escolares. El resultado está basado en un estudio de Reynolds \& Bartell (1984), que utiliza evaluaciones repetidas de grupos depresivos y no depresivos de adolescentes cada seis semanas, durante el año académico. Los resultados sugieren que hay adolescentes que permanecen deprimidos por extensos periodos de tiempo. En un estudio longitudinal realizado por Kovacs, Feinberg, CrouseNovak, Paulauskas, Pollock y Finkelstein (1984), se demostró que los desórdenes distímicos constituyen un problema real y de larga duración para algunos niños y adolescentes.

Estudios recientes enfatizan la necesidad de los especialistas en salud mental, de la evaluación de los síntomas depresivos en los adolescentes (Albert \& Beck, 1975; Reynolds, 1983; Reynolds \& Coats, 1986; Yanchyshyn \& Robbins, 1983).

Se ha creado una diversidad de instrumentos para la evaluación de los estados depresivos, sobre todo en adultos, tanto con fines clínicos como de investigación, pero esto no ha sucedido con la evaluación de niños y jóvenes. Como respuesta a esta carencia, entre otros esfuerzos, Kovacs (1979) desarrolló el inventario de depresión para niños (IDN), como una herramienta útil para la detección de este trastorno en menores de edad, instrumento que se encuentra actualmente en proceso de validación en algunos países. Esta tarea ha sido motivada principalmente por la necesidad que afrontan los profesionales de contar con instrumentos que agilicen y faciliten su trabajo.

En Latinoamérica son escasos los estudios abocados a la tarea de adaptar 
y/o desarrollar instrumentos psicométricos, que permitan evaluar la eficacia de los programas preventivos y de las intervenciones clínicas o servir como instrumentos diagnósticos. En Puerto Rico, Bernal, Bonilla y Santiago (1995) desarrollaron un estudio para determinar la confiablidad y validez de constructo del inventario de depresión de Beck (IDB) y de la lista de cotejos de síntomas-36 (LCS-36) en una muestra de 300 pacientes clínicos ambulatorios. El coeficiente alfa para el IDB fue de .8948 y para el LCS-36 de .9469 . Cuatro factores fueron hallados para el IDB: Actitudes negativas, cognitivoconductual, biológico y somático. En Cuba, Lugo, Louro y Bayarre (1998) validaron el inventario de Beck en una muestra de 144 sujetos depresivos y no depresivos, siendo los hallazgos congruentes con las dimensiones establecidas por los otros estudios sobre la validez del constructo de depresión.

En Costa Rica, el Instituto de Investigaciones Psicológicas (1996) realizó un estudio con jóvenes costarricenses entre 15 y 25 años; se utilizó un cuestionario adaptado del inventario de Beck para la evaluación de la depresión. Se reportaron niveles bajos de depresión y se señaló que hubo puntajes ligeramente altos en dos de las dimensiones de la depresión, la expresión de la ira y la percepción de problemas físicos. En Guatemala, Berganza y Aguilar (1992) realizaron un estudio en 339 adolescentes de 15 años, utilizando la escala de depresión para niños del Center for Epidemiological Studies, se halló que el $35,1 \%$ de los entrevistados tenía depresión y se encontró diferencias significativas según el sexo.

En otros países se han realizado diversas publicaciones con respecto a la depresión. A través de las investigaciones del National Institute Health Epidemiologic Catchment Area, Myers, Weissman y Tischler (1984) encontraron una prevalencia de depresión en adultos del $5,7 \%$ que evidencia que este trastorno es uno de los más comunes. En Estados Unidos y Nueva Zelandia, los índices de depresión mayor en prepúberes, han sido de $1,9 \%$ y 1,8\% respectivamente, (Kashani, McGee, Clarkson et al., 1983). Otro estudio, realizado en Estados Unidos, con 5.596 estudiantes, encontró una prevalencia de 1,9\% de depresión mayor (Whitaker, Johnson, Shaffer, Rapoport, Kalikow, Walsh, Davies, Bariman \& Dolinsky, 1990). Kovacs (1989), por ejemplo, encontró evidencia de que los trastornos afectivos entre los niños y adolescentes eran más persistentes de lo que se había creído hasta el momento del estudio. Hizo, además, referencia a las numerosas consecuencias negativas de estos trastornos, lo cual nuevamente demuestra la necesidad de mayor investigación en el tema. En Suecia, Larsson \& Melin (1992) utilizaron el inventario de Kovacs, encontrándose una prevalencia de depresión del 10\%. No se hallaron diferencias por región ni por 
edad, pero sí por sexo: las mujeres presentaron más síntomas depresivos que los varones. En Egipto, Abou Nazel, Fahmy, Younis, Seif el-Din, Abdel Fatah, Mokhtar \& Ayoub (1991) aplicaron el inventario de depresión para niños (IDN) a una muestra de 1.561 estudiantes y se detectó una prevalencia del $10,25 \%$, con una correlación muy alta entre los puntajes indicadores de depresión en los niños y sus madres. Los datos nacionales e internacionales presentados anteriormente sugieren que la depresión en niños y adolescentes es un problema frecuente.

Aunque generalmente las personas cercanas al adolescente no identifican las manifestaciones depresivas, suicidas o situaciones vivenciales precipitantes de estos problemas, los especialistas refieren que todos los jóvenes en esta condición viven crisis emocionales ante las cuales se sienten impotentes. En los diversos estudios realizados en adolescentes se describe un perfil del joven con problemas depresivos, el cual se caracteriza por una historia de psicopatología pasada y reciente, que puede incluir consumo de drogas, trastornos de ansiedad, conductas problemáticas y padecimientos somáticos, intentos de suicidio previos, actitud pesimista, autorreproche constante por los fracasos, imagen corporal negativa, baja autoestima, excesiva dependencia de otros, excesiva autocrítica, pobres mecanismos de defensa, escaso apoyo social de amigos y familiares y mayor probabilidad de con- sumo de tabaco en comparación con sus pares no deprimidos.

Otro estudio, realizado con estudiantes de colegios en Oregon, identificó varios factores de riesgo para el desarrollo de problemas depresivos, entre los más relevantes está el antecedente de depresiones previas, familias disfuncionales, bajo nivel educativo de los padres, situaciones estresantes y poco apoyo social, ansiedad, estado depresivo subclínico, conducta suicida, consumo de cigarrillos, baja autoestima y pobre imagen corporal, problemas académicos, excesiva autocrítica, escasa competencia intelectual y pobres habilidades de ajuste, discapacidad física o mala condición de salud física, excesiva dependencia interpersonal, relaciones conflictivas, especialmente con los padres, y muerte prematura de un padre. En general se ha encontrado que las personas que tienden a deprimirse viven en un ambiente estresado la mayor parte del tiempo.

Además de los factores anteriormente mencionados, se ha determinado que la alta prevalencia de depresión en una misma familia, sugiere un componente genético en el origen de este trastorno. En gemelos monocigóticos criados juntos la concordancia fue de un $76 \%$; en aquellos criados por separado fue de $67 \%$ y en gemelos dicigóticos fue de $19 \%$. Existe una probabilidad del $27 \%$ de que una persona tenga trastornos depresivos cuando lo tiene uno de sus padres, y del 50 al 74\% si lo tienen ambos (Shaffi \& Shaffi, 1995). 
Las investigaciones epidemiológicas sobre la sintomatología depresiva sugieren que la depresión es un serio problema de salud mental entre los adolescentes. Usando los puntajes de corte de la EDAR y del inventario de depresión de Beck (BDI) (Beck, Ward, Mendelson, Mock, \& Erlbaugh, 1961), se constató que del $12 \%$ al $18 \%$ de los adolescentes se encuentra en un nivel clínico de sintomatología depresiva (Reynolds, 1983). Sin embargo, esto no es una evidencia de un diagnóstico formal depresivo, pero sugiere que un nivel significativo de adolescentes demuestra niveles patológicos o distrés psicológico. En un estudio usando el BDI con 300 escolares, Kaplan, Hong y Weinhold (1984) estimaron que el 22\% de la muestra se encontraba entre los rangos de depresión leve a severa, mientras que Teri (1982) habla de que en una muestra de 568 adolescentes, el 32\% mostraron un rango de moderado a severo en el BDI. En un estudio de depresión en adolescentes de Albert y Beck (1975), se administró una forma revisada del BDI en escolares del sexto, séptimo y octavo grados de escuelas parroquiales en Filadelfia. Ellos reportaron que el $36,5 \%$ de los estudiantes obtuvieron resultados del nivel moderado al severo.

La presencia de desórdenes distímicos en adolescentes ha sido notada por Friedman, Clarkin, Corn, Aronoff, Hurt y Murphy (1982), quienes lo vieron como un serio desorden en adolescentes y que los podrían llevar a una depresión mayor. Éstos y otros estudios (Robbins, Alessi, Cook, Poznanski, \& Yanchyshyn, 1982) sugieren que niveles significativos de depresión están presentes entre los adolescentes, lo que apoya la necesidad de la evaluación de estos desórdenes.

En el Perú, Novara, Sotillo y Warthon (1983) estandarizaron y validaron el IDB en 178 pacientes, que acudían a consulta externa del Instituto de Salud Mental Honorio Delgado-Hideyo Noguchi. Conformaron cuatro subgrupos: pacientes con depresión, con trastornos psiquiátricos, con trastornos de ansiedad y sin trastorno mental. La validez concurrente arrojó una correlación de 0,75 entre los puntajes alcanzados en el IDB y la entrevista psiquiátrica.

Ugarriza (1998), en una investigación con el inventario de depresión de Beck en alumnos universitarios de Lima de los primeros ciclos, halló que el 40,5\% de 1.502 estudiantes de una universidad estatal, presentaban una depresión leve, lo que sin duda es causa de preocupación.

Son pocos los instrumentos especialmente diseñados para la evaluación de la depresión infantil, sin embargo, sólo se tiene referencias de la estandarización realizada por Reátegui (1994) con Children's Depresion Inventory (CDI), de Kovacs, con el fin de obtener el título de licenciada en psicología en la Universidad Peruana Cayetano Heredia y que es utilizado en algunos centros de salud. La muestra escolar estu- 
vo conformada por tres grupos de niños normales de tres niveles socioeconómi$\cos (n=570)$ entre 8 y 14 años, y un grupo de escolares con problemas de aprendizaje $(n=30)$ entre 7 y 15 años. Los estudios de validez indicaron que el CDI adaptado discriminaba entre el grupo depresivo y los grupos escolares. Además, según el análisis discriminante, se determinó que algunos ítems tienen mayor potencia discriminativa. Sin embargo, las diferencias entre el grupo clínico con otros diagnósticos y el grupo escolar del mismo nivel socioeconómico no fueron significativas. Las correlaciones con otras medidas de depresión, de autoconcepto, personalidad y rendimiento escolar, indicaron una sólida validez concurrente. El análisis factorial demostró una composición multifactorial para las dos muestras. Las correlaciones ítem-test fueron significativas, así como el coeficiente de confiabilidad, reflejando buena consistencia interna y confiabilidad testretest.

Por otro lado, las investigaciones que hacen alusión al tema de la depresión en el Perú son, en su mayoría, tesis que sustentan casos clínicos. Los estudios de las características psicométricas de las pruebas que miden depresión, así como otro tipo de investigaciones al respecto no han tenido mucha cabida en nuestro país hasta el momento.

De ahí que haya sido necesario adaptar y estandarizar, en nuestro medio, la Escala de Depresión para Adolescentes de Reynolds (EDAR) que mide síntomas asociados con la depresión tanto motor-vegetativos, somáticos e interpersonales, pero que no ha sido diseñada para brindar un diagnóstico de un desorden específico depresivo.

\section{SEVERIDAD DE LA SINTOMATOLOGía DEPRESIVA VERSUS DIAGNÓSTICO DE DEPRESIÓN}

En el estudio de la depresión existe una distinción entre el diagnóstico formal de la depresión como un desorden definido por un conglomerado de síntomas y la evaluación de la severidad de la sintomatología depresiva usando autorreportes. La evaluación de la profundidad o severidad de los síntomas depresivos, usando medidas de lápiz y papel como la EDAR, ofrece información útil sobre el nivel de la sintomatología depresiva experimentada por el individuo. A menudo, en esta aproximación un puntaje de corte será utilizado para operacionalizar un nivel clínico relevante de la sintomatología depresiva, pero no es un método útil para dar un diagnóstico de la depresión equivalente a los sistemas de clasificación reconocidas como el DSM-III. El diagnóstico de la depresión sólo puede ser dado eficazmente cuando se utilizan procedimientos de evaluación más amplios, que incorporen entrevistas de diagnósticos estructuradas como el SADS. Aunque ha habido intentos para derivar una información producto de una evaluación pro- 
funda de los procedimientos de entrevista diagnóstica (Endicott, Cohen, Nee, Fleiss, \& Saratakos, 1981), estos dos enfoques han sido, en general, mutuamente excluyentes.

Existen diferencias en resultados de estos enfoques. Cuando se usa la EDAR no es posible un diagnóstico formal de la depresión. Esta escala evalúa la severidad de la sintomatología depresiva mediante un puntaje de corte derivado empíricamente, que permite la descripción del nivel clínico relevante de la sintomatología depresiva en los adolescentes, pero que no es equivalente a un diagnóstico de depresión.

\section{Evaluación BASADA EN LA EDAR}

La EDAR es una medida de la sintomatología depresiva en los adolescentes y, como tal, es consistente con los que Rehm (1981) refiere como el "formato tradicional para la evaluación de la depresión". Como procedimiento de evaluación de la depresión es similar a la mayoría de medidas de autorreporte de lápiz y papel, con la diferencia de que es el único instrumento desarrollado y diseñado específicamente para su uso con adolescentes.

El formato de respuesta de la EDAR evalúa la frecuencia de ocurrencia o la duración de cada síntoma específico, siendo similar a la utilizada para medir la depresión en el adulto.

\section{OBJetivos}

- Adaptar y estandarizar la EDAR en una muestra representativa de alumnos de secundaria de la ciudad de Lima.

- Estudiar las diferencias de los niveles de depresión que presentan los alumnos adolescentes de secundaria de la ciudad de Lima, considerando las variables género y tipo de gestión escolar.

- Establecer la confiabilidad de la escala.

- Determinar la validez de constructo de la escala mediante el análisis factorial.

\section{MÉTODo}

En este estudio se ha empleado un sistema básico de muestreo de escuelas, para garantizar la representatividad por nivel socioeconómico y grados del nivel secundario en el ámbito de Lima metropolitana.

\section{Población}

La población escolar de secundaria de menores de Lima metropolitana, está integrada por aquellos adolescentes que estudian durante el día, en el turno de la mañana o de la tarde.

El universo de estudio se encuentra definido por la población escolar de secundaria de menores correspondientes a los 49 distritos de Lima metropolitana de los cinco niveles socioeconómicos (Instituto Nacional de Estadística e Informática, INEI, 1999) distribui- 
dos por grados, de acuerdo con la Unidad de Estadística del Ministerio de Educación (Med).

La población escolar a fines de 1998 para Lima metropolitana fue de 143.735 alumnos, de los cuales el $21,62 \%$ era de primero de secundaria, el $20,63 \%$ de segundo de secundaria, y el $19,92 \%, 19,39 \%$ y el $18,43 \%$ eran de tercero, cuarto y quinto de secundaria. Según el INEI (1999), la distribución poblacional por niveles socioeconómicos, a fines de 1998, fue de 6,21\% para el nivel socioeconómico alto, $16,73 \%$ para el nivel medio alto, $22,08 \%$ de nivel medio, $21,55 \%$ de nivel medio bajo, y el 33,43\% del bajo.

Estos porcentajes, así como su distribución para todas las combinaciones posibles por grado y nivel socioeconómico, se presentan en la tabla $\mathrm{N}^{\circ} 1$.

\section{Tamaño de la muestra}

Como ha sido necesaria la representación de los cinco niveles socioeconómicos y de los cinco grados, se obtiene 25 combinaciones posibles. En cada combinación se espera como mínimo un promedio de 60 estudiantes. Para lograr esto se consideró un tamaño de muestra de $25 \times 60=1.500$ evaluados para garantizar todas las posibles comparaciones deseadas. Este número de evaluados se ha distribuido proporcionalmente, considerando los porcentajes de la tabla $\mathrm{N}^{\mathrm{O}} 2$ como sigue y luego esta distribución de alumnos fue llevada a una distribución por colegios.

Se supuso que en las aulas hay aproximadamente 30 alumnos, y se consideró que se puede evaluar en una misma escuela todos los grados educativos, se tendría la distribución de escuelas por nivel socioeconómico dividiendo la cantidad de alumnos por nivel socioeconómico entre 30 x $5=150$.

De acuerdo con esto se tiene aproximadamente dos colegios de nivel socioeconómico medio alto, dos colegios de nivel socioeconómico medio, dos colegios de nivel socioeconómico medio bajo, y tres colegios de nivel socioeconómico bajo. Para el caso del nivel socioeconómico alto también se tiene

Tabla № 1

Distribución porcentual poblacional de alumnos en 1998, según estrato socioeconómico y grado escolar de acuerdo con el INEI (1999) y el Med (1999)

\begin{tabular}{lcccccc}
\hline Grado & Alto & Medio alto & Medio & Medio bajo & Bajo & \multicolumn{1}{c}{ Total } \\
\hline Primero & 1,34 & 3,62 & 4,77 & 4,66 & 7,23 & 21,62 \\
Segundo & 1,28 & 3,45 & 4,56 & 4,45 & 6,90 & 20,63 \\
Tercero & 1,24 & 3,33 & 4,40 & 4,29 & 6,66 & 19,92 \\
Cuarto & 1,20 & 3,24 & 4,28 & 4,18 & 6,48 & 19,39 \\
Quinto & 1,14 & 3,08 & 4,07 & 3,97 & 6,16 & 18,43 \\
\hline Total & 6,21 & 16,73 & 22,08 & 21,55 & 33,43 & 100,00 \\
\hline
\end{tabular}


Tabla № 2

Distribución muestral de alumnos en 1998 según estrato socioeconómico y grado escolar de acuerdo con el INEI (1999) y el Med (1999)

\begin{tabular}{lcccccc}
\hline Grado & Alto & Medio alto & Medio & Medio bajo & Bajo & Total \\
\hline Primero & 20 & 54 & 72 & 70 & 108 & 324 \\
Segundo & 19 & 52 & 68 & 67 & 104 & 309 \\
Tercero & 19 & 50 & 66 & 64 & 100 & 299 \\
Cuarto & 18 & 49 & 64 & 63 & 97 & 291 \\
Quinto & 17 & 46 & 61 & 60 & 92 & 276 \\
\hline Total & 93 & 251 & 331 & 323 & 501 & 1.500 \\
\hline
\end{tabular}

un colegio, porque el número de alumnos por aula suele ser menor a 30 . Esto corresponde, entonces, a un total de 10 escuelas.

\section{Procedimiento}

Para garantizar la representatividad de la muestra respecto de la población, el muestreo usado ha sido polietápico, porque comprende dos etapas sucesivas. En este caso, en una primera etapa se seleccionaron los distritos y en una segunda los colegios.

Selección de los distritos de Lima metropolitana

Con el plano de manzanas del INEI (1999), los distritos se han ubicado en un estrato socioeconómico (alto, medio alto, medio, medio bajo y bajo) sobre la base del porcentaje poblacional mayoritario existente según dicho estudio, lo que se presenta en la tabla $\mathrm{N}^{\circ} 3$.

De un total de 49 distritos de Lima metropolitana, se seleccionó el 15\%, es decir siete distritos, para luego deter- minar en ellos la muestra de escuelas. Esto significa un distrito dentro de cada nivel socioeconómico por cada siete de la lista. Según la tabla anterior se tiene un distrito medio alto, un distrito medio, y cuatro distritos bajos. Aunque el número sea menor, se ha considerado un distrito del nivel socioeconómico alto, y para el caso del nivel socioeconómico medio se ha tomado otro distrito ya seleccionado del nivel socioeconómico bajo, para así tener los siete considerados.

Los distritos elegidos fueron los siguientes:

- Alto: San Isidro

- Medio alto: Santiago de Surco

- Medio: Breña

- Medio bajo: Alguno de los distritos de nivel socioeconómico bajo

- Bajo: Ate-Vitarte, Comas, Villa María del Triunfo, Callao

\section{Selección de los centros educativos}

De acuerdo con el tamaño prefijado de escuelas y los distritos seleccionados en la etapa anterior, se obtuvo la 
Tabla № 3

Distribución porcentual de los distritos de Lima metropolitana según nivel socioeconómico

\begin{tabular}{|c|c|c|c|}
\hline Niveles & Distritos & $\mathrm{N}$ & $\%$ \\
\hline Alto & Miraflores, San Isidro & 2 & 4,1 \\
\hline Medio alto & $\begin{array}{l}\text { Barranco, Jesús María, La Molina, Lince, Magdalena, Pueblo Libre, } \\
\text { San Borja, San Miguel, Surco, La Punta }\end{array}$ & 10 & 20,4 \\
\hline Medio & $\begin{array}{l}\text { Breña, La Victoria, Lima, Rímac, San Luis, San Martín de Porres, } \\
\text { Surquillo, Bellavista, La Perla }\end{array}$ & 9 & 18,4 \\
\hline Medio bajo & Carmen de la Legua & 1 & 2,0 \\
\hline Bajo & $\begin{array}{l}\text { Ancón, Ate-Vitarte, Carabayllo, Cieneguilla, Comas, Chaclacayo, } \\
\text { Chorrillos, El Agustino, Independencia, Los Olivos, Lurigancho, Lurín, } \\
\text { Pachacámac, Puente Piedra, Pucusana, Punta Hermosa, Punta Negra, } \\
\text { San Bartolo, SJ. de Lurigancho, S.J de Miraflores, Santa Anita, Santa Mar } \\
\text { Santa Rosa, Villa El Salvador, Villa María del Triunfo, Callao, Ventanilla }\end{array}$ & $\begin{array}{l}\text { ría, } \\
27\end{array}$ & 55,1 \\
\hline
\end{tabular}

selección de la tabla $\mathrm{N}^{\circ} 4$. Para garantizar la representatividad por nivel socioeconómico se decidió tomar inicialmente la gestión de la escuela como indicador. Tomando en cuenta una distribución simétrica. Escuelas no estatales en los niveles alto y medio alto, y escuelas estatales en los niveles bajo y medio bajo, y mixtas en el nivel medio, como se expresa en la tabla $\mathrm{N}^{\circ} 4$.

\section{Definición de la muestra}

La selección de la muestra fue de carácter no probabilístico, ya que se realizó de manera intencional, proporcional al tamaño; de acuerdo a dos criterios de estratificación: estrato socioeconómico (INEI) y grado escolar (Ministerio de Educación) y bietápica porque se seleccionaron distritos en una primera etapa y escuelas en la segunda etapa.

En lo que respecta al género, no se consideró como criterio de estratificación, pero se buscó que la muestra fuese balanceada.

\section{Dominios del muestreo}

Para efectos de nuestro estudio, se consideraron los siguientes criterios:

\section{Tabla № 4}

\section{Características de las escuelas seleccionadas}

\begin{tabular}{ll}
\hline Nivel socioeconómico & \\
\hline Alto & 1 escuela no estatal de San Isidro \\
Medio alto & 2 escuelas no estatales de Santiago de Surco \\
Medio & 1 escuela no estatal y 1 escuela estatal de Breña \\
Medio bajo & 2 escuelas estatales de Ate-Vitarte, Comas, Villa María del Triunfo, Callao \\
Bajo & 3 escuelas estatales de Ate-Vitarte, Comas, Villa María del Triunfo, Callao \\
\hline
\end{tabular}


- Criterios de inclusión:

- Grupos escolares pertenecientes a escuelas mixtas de educación básica.

- Escuelas clasificables en diferentes niveles socioeconómicos. La clasificación se obtuvo según el monto promedio de la pensión de enseñanza mensual, la infraestructura educativa, y la ubicación de la escuela en el plano de manzanas de Lima metropolitana, según niveles socioeconómicos, INEI (1998).

- Edad entre 12 y 18 años.

- Nivel secundario: nivel educacional donde se consideran desarrolladas las capacidades relacionadas con los cuantificadores, según la estructura curricular básica de educación primaria de menores, Med (2000).

- Criterios de exclusión:

Relacionados con las características de la muestra:

- Fuera del rango de edad: alumnos que no pertenecen a la edad normativa del grado más un año. Por ejemplo, las edades normativas en el segundo grado de secundaria son de 13 a 14 años, y en el cuarto grado son de 15 a 16 años. Puede ocurrir "corta edad" cuando la edad del alumno es menor a la edad normativa para el grado, y puede ocurrir "extraedad" cuando la edad del alum- no es mayor a la edad normativa del grado.

- Falta de datos: cuestionarios sin datos de edad y/o sexo.

Relacionados con la respuesta psicológica:

- Respuesta omitida: cuestionarios con siete o más omisiones. Considerándose como omisión más de dos alternativas marcadas en un ítem.

- Pruebas que, según protocolo, sean inválidas.

- El observar patrones inusuales de respuesta, como el elegir la misma alternativa en casi todos los ítems.

- Las respuestas inconsistentes a los pares de ítems: 1 y 7 , y 9 y 12 , que son opuestos pero similares en contenido.

Teniendo en cuenta los criterios expuestos, se depuraron 2.507 protocolos de la muestra inicialmente seleccionada, quedando como muestra final 1.963 evaluados, tal como aparece en la tabla $\mathrm{N}^{\circ} 5$, en ella se observa un $54,8 \%$ de varones y $45,2 \%$ de mujeres. Existe un mayor porcentaje entre los 14 y 15 años, la muestra se distribuye de manera homogénea de segundo a quinto grados de secundaria. Los estratos medio y medio bajo están representados en un $40 \%$ y el bajo en un $31 \%$. Con respecto a la gestión del colegio, el 53,4\% son estatales y el $46,6 \%$ son particulares. 
Tabla № 5

Distribución de la muestra de estandarización

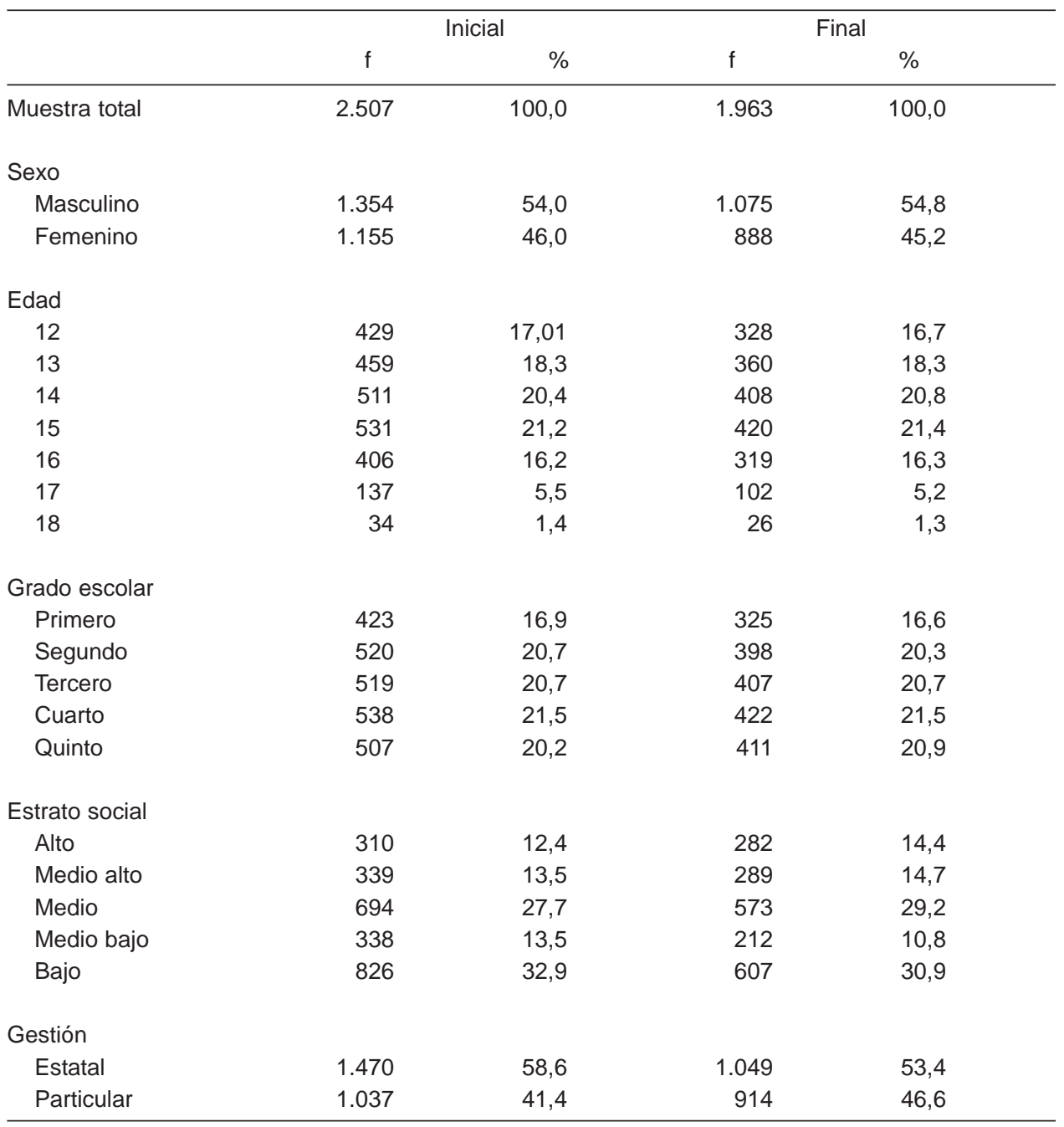

\section{Técnica de recolección de datos}

\section{Descripción}

La Escala de Depresión para Adolescentes de Reynolds (EDAR) es una prueba breve de fácil aplicación, desarrollada para evaluar la sintomatología depresiva en adolescentes cuyas edades fluctúan entre los 13 y 18 años, aunque puede ser usado en personas fuera de este rango de edades. Permite determinar los niveles clínicos relevantes de la sintomatología depresiva en adolescentes y es útil para identificar a las personas que denotan depresión 
dentro de las poblaciones escolares. Además, se la considera como una herramienta de recolección de datos para la investigación de la relación de la depresión con otros constructos y como medida de los resultados de algún tipo de tratamiento.

La EDAR puede ser aplicada individualmente o en pequeños grupos, y las instrucciones pueden ser expresadas oralmente cuando los adolescentes tienen problemas de lectura.

La escala contiene 30 ítems y utiliza un formato de respuesta tipo Likert de cuatro alternativas: casi nunca, rara vez, algunas veces y casi siempre. El adolescente debe elegir y marcar la alternativa con cuyo contenido se siente más identificado. Los ítems están escritos en tiempo presente para elicitar síntomas actuales. El formato de respuesta evalúa la frecuencia de los síntomas que son psicopatológicamente positivos del desorden depresivo. De los 30 ítems, siete son inconsistentes con la depresión: 1, 5, 10, 12, 23, 25 у 29. Estos reactivos se califican de modo inverso, es decir la respuesta "casi nunca" recibe un puntaje de 4; "rara vez": 3 puntos; "algunas veces": 2 puntos y "casi siempre": 1 punto.

\section{Selección de ítems}

La selección de ítems se basó en la sintomatología delineada del DSM-III para la depresión mayor y los desórdenes distímicos, así como también los síntomas adicionales del RDC, como es mostrado por el SADS, en ambas versiones para niños y adultos. La tabla $\mathrm{N}^{\circ}$ 6 ilustra los ítems de la EDAR, contenidos, la sintomatología de la depresión y sus fuentes; los ítems congruentes con los síntomas especificados por el criterio de Weinberg y la Escala de Depresión de Hamilton (Hamilton, 1960, 1967).

Un número de ítems se traslapan en el dominio general del síntoma, pero difiere en la especificidad en la cual evalúa el síntoma. Por ejemplo, el ítem 3 y el 6 son indicadores de que existe un déficit en las habilidades sociales y en las actividades socialmente reforzantes, de ahí que se traslapan en alguna medida. Sin embargo, el ítem 3 reporta la falta de interacción social mientras que el 6 es un indicador de la evitación de tal actividad. El traslape entre el ítem 1 ("me siento feliz") y en el ítem 7 ("me siento triste") es diferente y presentado así a propósito.

Estos ítems tienen que ver con los síntomas generales del estado de ánimo disfórico, que es un síntoma principal de la depresión. La importancia del estado de ánimo disfórico en la depresión justifica el mayor peso otorgado al considerar dos ítems referidos a este síntoma en el contenido de la EDAR.

La anhedonia también se considera dentro de esta conceptualización. Ambos síntomas son incorporados como criterios de inclusión primaria en el DSM-III para depresión mayor y desórdenes distímicos, y en el criterio de 
Tabla № 6

Ítems de la EDAR, sintomatología y fuentes asociadas

\begin{tabular}{|c|c|c|c|c|c|}
\hline Ítems & $\begin{array}{c}\text { EDAR } \\
\text { Sintomatología asociada }\end{array}$ & DSM-III R & $\mathrm{RDC}$ & $\begin{array}{c}\text { Fuentes* } \\
\text { Weinberg criterial }\end{array}$ & Hamilton \\
\hline 1 & Disforia & $x$ & $x$ & $\mathrm{X}$ & $\mathrm{X}$ \\
\hline 2 & Ansiedad escolar & & $x$ & & $x$ \\
\hline 3 & Soledad & & $\mathrm{X}$ & $\mathrm{X}$ & \\
\hline 4 & Devaluado por los padres & $x$ & $x$ & & $x$ \\
\hline 5 & Mérito propio, valía personal & $x$ & $x$ & $x$ & \\
\hline 6 & Aislamiento social & $x$ & $x$ & $x$ & \\
\hline 7 & Tristeza & $\mathrm{X}$ & $\mathrm{X}$ & $\mathrm{x}$ & $\mathrm{X}$ \\
\hline 8 & Llanto & $x$ & $x$ & $x$ & $x$ \\
\hline 9 & No apreciado, no valorado & $x$ & $x$ & $x$ & $x$ \\
\hline 10 & Anhedonia - pares & $x$ & $x$ & $x$ & \\
\hline 11 & Queja somática & $x$ & $x$ & $x$ & $x$ \\
\hline 12 & Bajo mérito personal & $x$ & $\mathrm{x}$ & $x$ & $x$ \\
\hline 13 & Abatimiento & & $x$ & & $x$ \\
\hline 14 & Daño a sí mismo & $\mathrm{x}$ & $\mathrm{X}$ & & $x$ \\
\hline 15 & Autoestima & $x$ & & & $x$ \\
\hline 16 & Irritabilidad & $x$ & $\mathrm{X}$ & & $x$ \\
\hline 17 & Pesimismo & & $x$ & & $x$ \\
\hline 18 & Fatiga & $x$ & $x$ & $x$ & $x$ \\
\hline 19 & Autorreproche & $x$ & $x$ & & $\mathrm{x}$ \\
\hline 20 & Desaprobarse a sí mismo & $x$ & $x$ & $x$ & $x$ \\
\hline 21 & Pena de sí mismo & $x$ & $\mathrm{X}$ & & \\
\hline 22 & Cólera & $x$ & $\mathrm{X}$ & $x$ & \\
\hline 23 & Reducción del habla & $x$ & $x$ & & $x$ \\
\hline 24 & Alteraciones del sueño & $\mathrm{x}$ & $\mathrm{X}$ & $\mathrm{x}$ & $\mathrm{x}$ \\
\hline 25 & Anhedonia - general & $x$ & $x$ & & $x$ \\
\hline 26 & Preocupación & & $x$ & & $x$ \\
\hline 27 & Queja somática & $x$ & $x$ & $x$ & $x$ \\
\hline 28 & Pérdida de interés & $x$ & $x$ & $x$ & $x$ \\
\hline 29 & Desórdenes del apetito & $x$ & $\mathrm{X}$ & $x$ & $x$ \\
\hline 30 & Desesperanza & $x$ & $x$ & & $x$ \\
\hline
\end{tabular}

* Fuentes de los síntomas:

DSM - III Manual Diagnóstico y Estadístico de los Trastornos Mentales. 3a. edición.

RDC: Schedule for Afecctive Disorders and Schizophenia - Adult versión (Endicott \& Spitzer, 1978) and Child version (K-SADS: Puig - Antich et al.,1980).

Hamilton $(1960,1967) 24$ ítems (NIMH) version modified to accommodate school as work setting.

Weinberg especifica la presencia del estado de ánimo disfórico y el pensamiento de no ser apreciado o valorado. Este último se repite a través de varios ítems de la EDAR.

\section{Administración, puntajes $e$ interpretación}

La administración puede ser individual o en grupos pequeños; esta forma de la EDAR de calificación manual, re- 
quiere de entre cinco a diez minutos para completar el cuestionario. Para evitar la posibilidad de influir en el estado de ánimo de los evaluados, la escala no lleva el título de "depresión", sino "sobre mí mismo". Es suficiente indicar que la EDAR es un cuestionario diseñado para evaluar los sentimientos de los adolescentes acerca de ellos mismos y de cosas en general.

\section{INTERPRETACIÓN DE LA PRUEBA}

\section{Puntaje total y puntaje de corte de la EDAR}

El puntaje de corte de la EDAR puede ser usado para juzgar la severidad del síntoma depresivo. Se debe identificar al adolescente cuyo puntaje directo se encuentra por encima de 76 en la EDAR, y luego ser evaluado de modo más profundo, con el propósito de diagnosticar potencialmente una psicopatología significativa.

La validación clínica del puntaje de corte fue probada rigurosamente en dos investigaciones, cuyos resultados fundamentan el uso de este puntaje de corte para distinguir entre adolescentes deprimidos de los que no lo están (Reynolds, 1987).

Los ítems críticos en la EDAR son aquéllos que discriminan a los adolescentes deprimidos desde un punto de vista clínico y de aquéllos que no lo son. Los ítems críticos son el 6, 14, 20, 26, 29 y el 30.

\section{Indicadores de validez de la EDAR}

Para considerar inválido un protocolo de la EDAR se debe tener en cuenta lo siguiente:

- Seis o más omisiones en un protocolo invalidan la prueba.

- Respuestas que siguen un mismo patrón, por ejemplo responder igual a todos los ítems.

- Respuestas inconsistentes a los pares de ítems 1 y 7 y el 9 y 12; que son opuestos pero lógicamente similares.

Hay que tener presente al interpretar el puntaje total de la EDAR, que otros factores puedan influir en la respuesta del adolescente, tales como una enfermedad, problemas para entender los ítems o una combinación de factores que pueden llevar a invalidar el protocolo.

\section{RESUltados}

En la tabla $\mathrm{N}^{\circ} 7$ se presentan las medias, desviaciones estándares para la muestra total de estandarización y para las submuestras considerando el sexo y la gestión del colegio. Además se computaron los tests $t$ para muestras independientes, señalándose las diferencias significativas para las comparaciones de medias correspondientes a los 30 ítems de la EDAR y al puntaje total. Con relación al puntaje total las puntuaciones medias totales son más elevadas y presenta una mayor variabilidad en el grupo de mujeres $($ Media $=61.55>57,19)$ 
y según la gestión, los alumnos de los colegios estatales denotan una mayor depresión que los particulares. (62.33 > 61.55). En la misma tabla se observa que en 20 de 30 ítems los varones denotan una media más elevada y 24 de 30 ítems de la escala de depresión, los adolescentes de colegios estatales presentan puntajes medios más altos y estadísticamente significativos.

Tabla № 7

Media de ítems para la muestra total y el puntaje total, por sexo y tipo de gestión

\begin{tabular}{|c|c|c|c|c|c|c|c|c|c|c|}
\hline \multirow{3}{*}{ Ítems } & \multirow{2}{*}{\multicolumn{2}{|c|}{$\begin{array}{c}\text { Muestra } \\
\text { Total } \\
\mathrm{N}=1963\end{array}$}} & \multicolumn{4}{|c|}{ Sexo } & \multicolumn{4}{|c|}{ Gestión } \\
\hline & & & \multicolumn{2}{|c|}{$\begin{array}{r}\text { Varón } \\
\mathrm{N}=1075\end{array}$} & \multicolumn{2}{|c|}{$\begin{array}{r}\text { Mujer } \\
\mathrm{N}=888\end{array}$} & \multicolumn{2}{|c|}{$\begin{array}{r}\text { Estatal } \\
\mathrm{N}=1049\end{array}$} & \multicolumn{2}{|c|}{$\begin{array}{l}\text { Particular } \\
\mathrm{N}=914\end{array}$} \\
\hline & $M$ & D.S. & M & D.S. & M & D.S. & M & D.S. & M & D.S. \\
\hline Disforia & 1.56 & .67 & 1.54 & .64 & 1.58 & .71 & $1.67^{\star \star}$ & .70 & 1.43 & .62 \\
\hline Ansiedad escolar & 3.21 & .84 & $3.09^{* *}$ & .86 & 3.35 & .80 & $3.24^{*}$ & .88 & 3.17 & .80 \\
\hline Soledad & 2.16 & .98 & $2.04^{* *}$ & .95 & 2.30 & 1.0 & $2.28^{* \star}$ & 1.01 & 2.02 & .92 \\
\hline Devaluado por padres & 1.72 & .97 & $1.62^{* *}$ & .93 & 1.84 & 1.0 & $2.01^{* *}$ & 1.06 & 1.39 & .74 \\
\hline Valía personal & 2.08 & .95 & 2.09 & .92 & 2.08 & .98 & $2.21^{* *}$ & 1.02 & 1.94 & .84 \\
\hline Aislamiento & 1.70 & .87 & 1.69 & .86 & 1.72 & .87 & $1.75^{\star}$ & .90 & 1.65 & .82 \\
\hline Tristeza & 2.23 & .92 & $2.05^{\star \star}$ & .87 & 2.44 & .93 & $2.36^{\star \star}$ & .94 & 2.07 & .87 \\
\hline Llanto & 2.03 & .95 & $1.72^{* *}$ & .83 & 2.41 & .95 & $2.18^{* *}$ & 1.00 & 1.86 & .86 \\
\hline No apreciado & 1.75 & .89 & $1.63^{* *}$ & .81 & 1.89 & .96 & $1.94^{* *}$ & .95 & 1.54 & .76 \\
\hline Anhedonia pares & 1.48 & .76 & $1.44^{*}$ & .69 & 1.54 & .83 & $1.64^{* *}$ & .85 & 1.31 & .59 \\
\hline Queja somática & 1.70 & .83 & 1.67 & .80 & 1.74 & .85 & $1.81^{* *}$ & .88 & 1.58 & .79 \\
\hline Bajo mérito personal & 1.60 & .82 & 1.57 & .78 & 1.64 & .86 & $1.77^{\star *}$ & .89 & 1.41 & .67 \\
\hline Abatimiento & 1.65 & .89 & $1.57^{* *}$ & .84 & 1.75 & .94 & $1.74^{* *}$ & .95 & 1.54 & .81 \\
\hline Daño a sí mismo & 1.33 & .70 & $1.28^{\star *}$ & .64 & 1.40 & .76 & $1.42^{* *}$ & .77 & 1.24 & .59 \\
\hline Autoestima & 1.90 & .91 & 1.89 & .90 & 1.91 & .93 & $1.99^{* *}$ & .93 & 1.79 & .89 \\
\hline Irritabilidad & 2.17 & .90 & $2.13^{*}$ & .90 & 2.22 & .90 & $2.22^{*}$ & .92 & 2.12 & .87 \\
\hline Pesimismo & 2.31 & 1.01 & $2.22^{* *}$ & .97 & 2.41 & 1.03 & $2.41^{* *}$ & 1.04 & 2.19 & .96 \\
\hline Fatiga & 2.28 & .92 & 2.26 & .91 & 2.30 & .93 & 2.31 & .94 & 2.23 & .90 \\
\hline Autorreproche & 1.74 & .87 & 1.76 & .88 & 1.71 & .86 & $1.85^{\star *}$ & .92 & 1.60 & .79 \\
\hline Desaprobarse a sí mismo & 1.50 & .83 & $1.43^{* *}$ & .76 & 1.59 & .89 & $1.65^{* *}$ & .91 & 1.34 & .67 \\
\hline Compasión de sí mismo & 1.61 & .87 & 1.58 & .85 & 1.64 & .90 & $1.80^{* *}$ & .95 & 1.39 & .72 \\
\hline Cólera & 2.72 & .84 & $2.64^{* *}$ & .87 & 2.81 & .79 & 2.73 & .88 & 2.71 & .78 \\
\hline Reducción de la comunicación & 1.58 & .83 & $1.63^{*}$ & .85 & 1.53 & .81 & $1.67^{* *}$ & .92 & 1.48 & .71 \\
\hline Alteraciones del sueño & 1.76 & .98 & $1.74^{*}$ & .96 & 1.78 & .01 & 1.79 & 1.01 & 1.72 & .95 \\
\hline Anhedonia general & 1.51 & .79 & $1.45^{\star *}$ & .72 & 1.58 & .86 & $1.68^{* *}$ & .90 & 1.31 & .59 \\
\hline Preocupado & 2.50 & .94 & $2.39^{* *}$ & .93 & 2.63 & .93 & $2.55^{\star *}$ & .98 & 2.44 & .88 \\
\hline Queja somática & 1.85 & .94 & $1.71^{* *}$ & .87 & 2.01 & .99 & $1.98^{* *}$ & .99 & 1.69 & .84 \\
\hline Aburrimiento & 2.34 & .93 & $2.24^{* *}$ & .92 & 2.45 & .93 & 2.37 & .96 & 2.31 & .89 \\
\hline Trastornos del apetito & 1.60 & .84 & $1.53^{\star \star}$ & .79 & 1.69 & .90 & 1.57 & .87 & 1.64 & .81 \\
\hline Desesperanza & 2.00 & .99 & 1.96 & .96 & 2.05 & .02 & $2.23^{\star *}$ & 1.03 & 1.75 & .87 \\
\hline Puntaje total & 59.16 & 11.61 & $57.19^{* *}$ & 10.31 & 61.55 & 12.62 & $62.33^{* *}$ & 11.50 & 55.54 & 0.65 \\
\hline
\end{tabular}

${ }^{*} p<.05$

${ }^{* *} p<.01$ 


\section{Confiabilidad}

\section{Consistencia interna}

La consistencia interna fue obtenida mediante el coeficiente alpha de Cronbach (1951). Se estimó la confiabilidad para la muestra de estandarización y para varias submuestras por grado, sexo y gestión (tabla $\mathrm{N}^{\circ}$ 8). El alfa para toda la muestra total de estandarización fue de .87. Los coeficientes de consistencia interna más altos, entre .86 y .90 , se registraron en los diferentes grados escolares de secundaria de los colegios particulares; y los más bajos en los varones de primero a tercero de secundaria, de .76 a .79 .

El análisis de los coeficientes alfa de Cronbach para el puntaje total, eliminando el ítem correspondiente de la EDAR, considerando la muestra total y submuestras se presenta en la tabla $\mathrm{N}^{\mathrm{O}}$ 9 , apreciándose correlaciones altas, por encima de .84 para los 30 ítems.
La confiabilidad determinada por el método de división por mitades para la muestra total de estandarización fue de .85 , corregido por la fórmula de Spearman Brown. Por el mismo procedimiento la consistencia interna para las submuestras de mujeres y de colegios particulares fue de .86, tal como se aprecia en la tabla $\mathrm{N}^{\mathrm{o}} 10$, también son indicadores confiables del nivel general de la sintomatología de la depresión.

En un análisis de la confiabilidad test-retest desarrollado por los estudiantes del curso de Psicometría II, de la Universidad de Lima, utilizaron el procedimiento retest con la EDAR transcurrido un periodo de dos semanas, en una muestra de 142 alumnos del Colegio Estatal $N^{\circ} 1230$. Obtuvieron un coeficiente de confiabilidad de .71 para los grados primero y segundo, compuesto por 60 adolescentes, y de .89 para el grupo de tercero a quinto de secundaria, integrado por 82 adolescentes. Se puede observar en la tabla

Tabla № 8

Confiabilidad por consistencia interna mediante el coeficiente alfa de Cronbach para el puntaje total de la EDAR para la muestra total de estandarización y submuestras

\begin{tabular}{|c|c|c|c|c|c|c|c|c|c|c|}
\hline \multirow[t]{3}{*}{ Grado } & \multicolumn{6}{|c|}{ Sexo } & \multicolumn{4}{|c|}{ Gestión } \\
\hline & \multicolumn{2}{|c|}{ Muestra total } & \multicolumn{2}{|c|}{ Varones } & \multicolumn{2}{|c|}{ Mujeres } & \multicolumn{2}{|c|}{ Estatal } & \multicolumn{2}{|c|}{ Particular } \\
\hline & $\mathrm{N}$ & Alfa & $\mathrm{N}$ & Alfa & $\mathrm{N}$ & Alfa & $\mathrm{N}$ & Alfa & $\mathrm{N}$ & Alfa \\
\hline 1 & 325 & .85 & 242 & .76 & 128 & .83 & 166 & .81 & 159 & .86 \\
\hline 2 & 398 & .86 & 282 & .79 & 180 & .86 & 185 & .81 & 213 & .87 \\
\hline 3 & 407 & .89 & 275 & .77 & 184 & .90 & 221 & .88 & 186 & .88 \\
\hline 4 & 422 & .88 & 283 & .82 & 202 & .88 & 232 & .85 & 190 & .88 \\
\hline 5 & 411 & .89 & 271 & .83 & 194 & .90 & 245 & .87 & 166 & .90 \\
\hline Total & 1963 & .87 & 1075 & .85 & 888 & .89 & 1049 & .85 & 914 & .88 \\
\hline
\end{tabular}


Tabla № 9

Análisis de los coeficientes alfa de Cronbach para el puntaje total eliminando el ítem de la EDAR para la muestra total de estandarizacion y submuestras

\begin{tabular}{|c|c|c|c|c|c|}
\hline \multirow[t]{2}{*}{ İtem } & $\begin{array}{c}\text { Muestra } \\
\text { total }\end{array}$ & Varones & Mujeres & Estatal & Particular \\
\hline & $(\mathrm{N}=1963)$ & $(\mathrm{N}=1075)$ & $(\mathrm{N}=808)$ & $(\mathrm{N}=1049)$ & $(\mathrm{N}=914)$ \\
\hline Disforia & .8683 & .8400 & .8856 & .8446 & .8760 \\
\hline Ansiedad escolar & .8768 & .8525 & .8924 & .8532 & .8853 \\
\hline Soledad & .8677 & .8408 & .8846 & .8436 & .8752 \\
\hline Devaluado por padres & .8696 & .8419 & .8870 & .8466 & .8781 \\
\hline Valía personal & .8721 & .8457 & .8879 & .8502 & .8785 \\
\hline Aislamiento & .8704 & .8417 & .8878 & .8468 & .8772 \\
\hline Tristeza & .8659 & .8377 & .8838 & .8415 & .8736 \\
\hline Llanto & .8669 & .8405 & .8840 & .8421 & .8754 \\
\hline No apreciado & .8655 & .8379 & .8829 & .8405 & .8743 \\
\hline Anhedonia pares & .8738 & .8469 & .8907 & .8515 & .8817 \\
\hline Queja somática & .8709 & .8442 & .8872 & .8466 & .8795 \\
\hline Bajo mérito personal & .8680 & .8411 & .8844 & .8433 & .8771 \\
\hline Abatimiento & .8680 & .8395 & .8858 & .8429 & .8763 \\
\hline Daño a sí mismo & .8695 & .8430 & .8860 & .8446 & .8781 \\
\hline Autoestima & .8710 & .8427 & .8882 & .8489 & .8771 \\
\hline Irritabilidad & .8700 & .8409 & .8878 & .8464 & .8766 \\
\hline Pesimismo & .8682 & .8412 & .8849 & .8443 & .8754 \\
\hline Fatiga & .8714 & .8430 & .8887 & .8481 & .8780 \\
\hline Autorreproche & .8705 & .8418 & .8876 & .8483 & .8769 \\
\hline Desaprobarse a sí mismo & .8660 & .8380 & .8834 & .8413 & .8743 \\
\hline Compasión de sí mismo & .8673 & .8385 & .8847 & .8440 & .8749 \\
\hline Cólera & .8718 & .8436 & .8894 & .8480 & .8785 \\
\hline Reducción de la comunicación & .8758 & .8491 & .8917 & .8540 & .8829 \\
\hline Alteraciones del sueño & .8733 & .8455 & .8903 & .8479 & .8823 \\
\hline Anhedonia general & .8739 & .8470 & .8909 & .8520 & .8817 \\
\hline Preocupado & .8691 & .8418 & .8861 & .8447 & .8762 \\
\hline Queja somática & .8706 & .8443 & .8875 & .8468 & .8789 \\
\hline Aburrimiento & .8697 & .8422 & .8869 & .8447 & .8773 \\
\hline Trastornos del apetito & .8763 & .8501 & .8930 & .8514 & .8847 \\
\hline Desesperanza & .8671 & .8402 & .8833 & .8437 & .8749 \\
\hline
\end{tabular}

Tabla № 10

Análisis de la confiabilidad de la EDAR por el método de mitades para la muestra total de estandarización y submuestras

\begin{tabular}{lccccc}
\hline & Muestra & \multicolumn{2}{c}{ Sexo } & \multicolumn{2}{c}{ Gestión } \\
total & Varones & Mujeres & Estatal & Particular \\
& $(\mathrm{N}=1963)$ & $(\mathrm{N}=1075)$ & $(\mathrm{N}=888)$ & $(\mathrm{N}=1049)$ & $(\mathrm{N}=914)$ \\
\hline Mitades & .7437 & .7088 & .7651 & .6983 & .7674 \\
Spearman-Brown & .8530 & .8296 & .8669 & .8223 & .8684 \\
\hline
\end{tabular}


$\mathrm{N}^{\mathrm{o}} 11$, que las medias entre el test y el retest disminuyeron para ambos grupos.

En general los resultados de los estudios de la confiabilidad apoyan a la EDAR como una medida confiable.
La validez de contenido, examinada a través de la congruencia del ítem con la sintomatología clínica específica (tabla $\mathrm{N}^{\circ} 6$ ), permite apreciar que los 30 ítems de la EDAR se refieren a síntomas específicos de la depresión, e incluyen aquéllos señalados por la American

Tabla № 11

Medias para los puntajes totales y desviación estándar alcanzados en el test, retest de la EDAR en el colegio № 1230

\begin{tabular}{lccccc}
\hline Periodo & Grado & $\mathrm{N}$ & $\mathrm{M}$ & $\mathrm{DS}$ & $\mathrm{rx}$ \\
\hline Retest & 1 y 2 & 60 & 62,15 & 15,74 & .71 \\
(2 semanas) & 3,4 y 5 & 82 & 61,42 & 12,45 & .89 \\
\hline
\end{tabular}

\section{Validación de la EDAR}

Ha sido desarrollada para brindar un diagnóstico válido y confiable acerca de la gravedad de la sintomatología depresiva en los adolescentes. Con el propósito de validar la EDAR como una medida de la sintomatología depresiva en los adolescentes, se han llevado a cabo múltiples investigaciones en el extranjero durante un periodo de seis años, utilizándose diferentes procedimientos para probar la validez. Los resultados sobre la validez de contenido, concurrente y de constructo, esta última evaluada mediante la validez convergente, discriminante y factorial. La validez clínica demostrada por la sensibilidad de los reactivos para evaluar la eficacia del tratamiento y del diagnóstico, se reportan en el manual profesional de la escala de depresión para adolescentes de Reynolds (1987).
Psychiatric Association (APA) en el DSM -III, así como otros síntomas de depresión derivados de la investigación del criterio diagnóstico (RDC) e investigado por Carlson y Stroeber, en 1979, en su estudio de depresión unipolar en adolescentes.

Algunos ítems reflejan los componentes descriptivos de la depresión, por ejemplo síntomas cognitivos: "Siento que nada de lo que haga ayuda"; Síntoma psicomotor: "Me siento cansado" y déficit interpersonal: "Siento que a nadie le importo".

En el presente estudio las correlaciones ítem-escala total demuestran la consistencia interna del ítem con la suma de los otros ítems, lo que se aprecia en la tabla $\mathrm{N}^{\mathrm{o}} 12$ para la muestra total $\mathrm{y}$ las diferentes submuestras según sexo y tipo de gestión del colegio.

Se observa que todas las correlaciones 
Tabla № 12

Correlaciones de Pearson Ítem-puntaje total, para la muestra total de estandarización y submuestras

\begin{tabular}{lccccc}
\hline \multicolumn{1}{c}{ Ítems } & Muestra total & Varones & Mujeres & Estatal & Particular \\
\hline Disforia & .570 & .553 & .595 & .523 & .570 \\
Ansiedad escolar & .178 & .115 & .200 & .187 & .153 \\
Soledad & .557 & .491 & .601 & .520 & .583 \\
Devaluado por padres & .499 & .465 & .516 & .443 & 469 \\
Valía personal & .404 & .354 & .468 & .333 & .457 \\
Aislamiento & .333 & .327 & .348 & .288 & .391 \\
Tristeza & .626 & .585 & .638 & .590 & .646 \\
Llanto & .595 & .509 & .634 & .571 & .590 \\
No apreciado & .645 & .588 & .675 & .618 & .630 \\
Anhedonia pares & .296 & .267 & .308 & .247 & .259 \\
Queja somática & .434 & .387 & .480 & .426 & .403 \\
Bajo mérito personal & .557 & .485 & .626 & .536 & .520 \\
Abatimiento & .539 & .523 & .541 & .535 & .530 \\
Daño a sí mismo & .504 & .422 & .563 & .512 & .466 \\
Autoestima & .425 & .429 & .437 & .346 & .502 \\
Irritabilidad & .474 & .493 & .461 & .440 & .531 \\
Pesimismo & .543 & .486 & .588 & .499 & .588 \\
Fatiga & .422 & .435 & .419 & .384 & .487 \\
Autorreproche & .440 & .454 & .457 & .362 & .504 \\
Desaprobarse a sí mismo & .634 & .587 & .668 & .604 & .646 \\
Compasión de sí mismo & .569 & .551 & .597 & .506 & .599 \\
Cólera & .393 & .405 & .368 & .375 & .453 \\
Reducción de la comunicación & .222 & .234 & .246 & .184 & .225 \\
Alteraciones del sueño & .359 & .357 & .366 & .385 & .338 \\
Anhedonia general & .293 & .263 & .301 & .437 & .261 \\
Preocupado & .512 & .466 & .540 & .236 & .551 \\
Queja somática & .461 & .398 & .486 & .494 & .445 \\
Aburrimiento & .489 & .454 & .509 & .493 & .513 \\
Trastornos del apetito & .203 & .163 & .211 & .251 & .191 \\
Desesperanza & .587 & .520 & .658 & .529 & .599 \\
\hline & & & & & \\
\hline
\end{tabular}

son significativas al nivel de .001. Veintidós de 30 ítems tienen correlaciones mayores que $.40 \mathrm{y}$ lo mismo ocurre en el grupo de mujeres.

En los colegios particulares el número de ítems sobre la base del mismo criterio se incrementa a 23 ítems significativos. Los resultados, basados en la mues- tra de estandarización, demuestran que sólo 4 ítems tienen coeficientes por debajo de .30 .

Para propósitos prácticos, el contenido de la EDAR muestra los síntomas de depresión incluidos en la revisión propuesta por el DSM-III, los que también aparecen en el DSM-IV. 


\section{Análisis factorial}

En la construcción de la escala de depresión para adolescentes se siguió un procedimiento de muestreo de contenido, donde los ítems fueron desarrollados basándose en su convergencia con la sintomatología clínica específica, no habiéndose hipotetizado factores discretamente mensurables basados en un constructo teórico de la depresión.

En el presente estudio, con la finalidad de evaluar la viabilidad del análisis factorial para determinar la validez de constructo de la EDAR, se emplearon dos pruebas, cuyos resultados se aprecian en la tabla $\mathrm{N}^{\circ}$ 13: la medida de Kaiser-Meyer y Olkin (K-M-O) y el test de esfericidad de Barlett. Para la primera, que es una medida de adecuación de la muestra, el puntaje fue de .927 significativo al $.01 \mathrm{y}$ para el segundo se halló un valor de 13100.207. Estos puntajes permitieron continuar con el análisis.

El análisis de factores de la EDAR brinda un insight acerca de la estructura factorial subyacente de la escala, permitiendo además un examen posthoc de las relaciones de los ítems. Se ha utilizado un análisis de factores de componentes principales con estimación de comunalidades y una rotación ortogonal a través del método Varimax.

Por medio del método de análisis de los componentes principales se estimaron los factores de la Escala de Depresión para Adolescentes de Reynolds en la muestra total.
Tabla № 13

Medida de Kaiser-Meyer-Olkin de adecuación de la muestra de estudiantes universitarios de secundaria de Lima $(N=1963)$ y test de esfericidad de Barlett

\begin{tabular}{lcr}
\hline K-M-O & Test de Bartlett & \multicolumn{1}{c}{ Significación } \\
\hline .927 & 13100.207 & .000 \\
\hline
\end{tabular}

Figura № 1

Muestra total

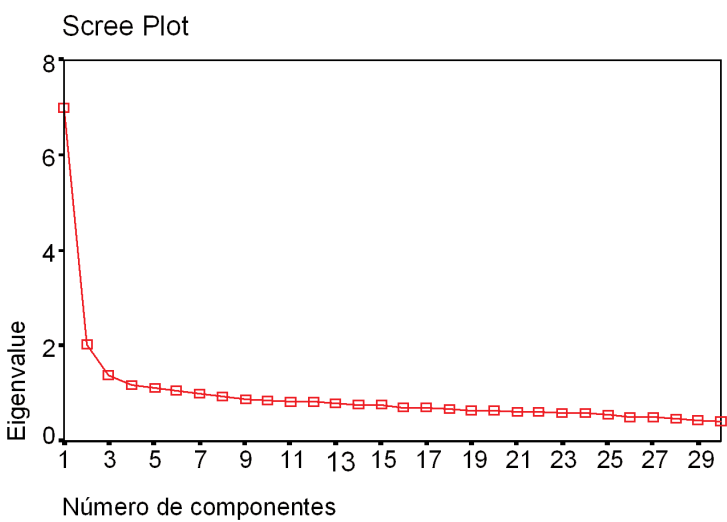

Se observa en la tabla $\mathrm{N}^{\circ} 14$ que el $e i$ genvalue para el factor 1 fue de 6.989, que representa un porcentaje de varianza explicada de 23.296. La mayoría de los ítems con pesos factoriales superiores a .30 (25 de 30$)$ cargaban sobre el primer factor, como se esperaba en una medida homogénea de la depresión como la EDAR.

Un scree test (Cattell, 1966), para determinar la significación de los factores, como aparece en la figura $\mathrm{N}^{\mathrm{o}} 1$, sugiere un primer factor consistente con el modelo del dominio de depresión que predice la EDAR. 
Tabla № 14

Pesos factoriales no rotados del primer factor obtenidos en el análisis de los componentes principales de los ítems de la EDAR en la muestra total de estandarización y submuestras por sexo.

\begin{tabular}{|c|c|c|c|c|}
\hline $\mathrm{N}^{\circ}$ & Ítems & $\begin{array}{c}\text { Muestra total } \\
N=1963\end{array}$ & $\begin{array}{c}\text { Varones } \\
\mathrm{N}=1075\end{array}$ & $\begin{array}{l}\text { Mujeres } \\
\mathrm{N}=888\end{array}$ \\
\hline 1 & Disforia & .672 & .564 & .605 \\
\hline 2 & Ansiedad escolar & .668 & & \\
\hline 3 & Soledad & .645 & .495 & .620 \\
\hline 4 & Devaluado por padres & .602 & .478 & .512 \\
\hline 5 & Valía personal & .598 & .325 & .455 \\
\hline 6 & Aislamiento & .598 & .461 & .452 \\
\hline 7 & Tristeza & .581 & .604 & .663 \\
\hline 8 & Llanto & .576 & .519 & .650 \\
\hline 9 & No apreciado & .569 & .619 & .701 \\
\hline 10 & Anhedonia pares & .569 & & \\
\hline 11 & Queja somática & .550 & .361 & .482 \\
\hline 12 & Bajo mérito personal & .528 & .489 & .644 \\
\hline 13 & Abatimiento & .506 & .569 & .576 \\
\hline 14 & Daño a sí mismo & .504 & .455 & .577 \\
\hline 15 & Autoestima & .482 & .435 & .436 \\
\hline 16 & Irritabilidad & .475 & .500 & .456 \\
\hline 17 & Pesimismo & .453 & .491 & .597 \\
\hline 18 & Fatiga & .448 & .422 & .396 \\
\hline 19 & Autorreproche & .443 & .471 & .468 \\
\hline 20 & Desaprobarse a sí mismo & .428 & .630 & .695 \\
\hline 21 & Compasión de sí mismo & .425 & .582 & .623 \\
\hline 22 & Cólera & .404 & .405 & .371 \\
\hline 23 & Reducción de la comunicación & .394 & & \\
\hline 24 & Alteraciones del sueño & .385 & .334 & .345 \\
\hline 25 & Anhedonia general & & & \\
\hline 26 & Preocupado & & .454 & .539 \\
\hline 27 & Queja somática & & .374 & .467 \\
\hline 28 & Aburrimiento & & .445 & .502 \\
\hline 29 & Trastornos del apetito & & & \\
\hline 30 & Desesperanza & .337 & .522 & .675 \\
\hline \multicolumn{2}{|c|}{ Eigenvalue } & 6.989 & 6.076 & 7.767 \\
\hline \multicolumn{2}{|c|}{$\%$ Varianza explicada } & 23.296 & 20.254 & 25.889 \\
\hline
\end{tabular}

Nota: Sólo se han incluido los pesos factoriales mayores que 0.30

En la tabla $N^{\circ} 15$ se presentan los pesos factoriales rotados de los ítems de la EDAR para la muestra total de estandarización, que son validados para cada factor, empleándose un índice de discriminación significativo mayor que .40. La solución factorial dio lugar a seis factores obtenidos por rotación ortogonal, mediante el método Varimax presentando los siguientes 
eigenvalues (lambda): 4.013, 3.033, $1.983,1.903,1.561$ y 1.243 que dan cuenta de una varianza total explicada de 45.786 .

El primer factor rotado parece representar un factor general de desmoralización con sentimientos de desesperan- za, no sentirse apreciado, bajo mérito personal, abatimiento, soledad y llanto; el segundo factor comprende ítems que se caracterizan por expresiones emocionales vinculadas a la cólera, irritabilidad, desánimo, aburrimiento, tristeza y fatiga; el tercer factor sugiere anhe-

Tabla № 15

Pesos factoriales rotados de los ítems de la EDAR mediante el método Varimax para la muestra total de estandarización $(\mathrm{N}=1963)$

\begin{tabular}{|c|c|c|c|c|c|c|c|c|}
\hline No. & Ítems & F 1 & $\mathrm{~F} 2$ & F 3 & $\mathrm{~F} 4$ & F 5 & $\mathrm{~F} 6$ & h2 \\
\hline 20 & Desaprobarse a sí mismo & .661 & & & & & & .583 \\
\hline 9 & No apreciado & .641 & & & & & & .583 \\
\hline 12 & Bajo mérito personal & .638 & & & & & & .516 \\
\hline 4 & Devaluado por padres & .598 & & & & & & .425 \\
\hline 13 & Abatimiento & .548 & & & & & & .441 \\
\hline 14 & Daño a sí mismo & .535 & & & & & & .398 \\
\hline 1 & Disforia & .515 & & & & & & .485 \\
\hline 30 & Desesperanza & .495 & & & & & & .417 \\
\hline 3 & Soledad & .453 & & & & & & .390 \\
\hline 8 & Llanto & .439 & . & & & & & .506 \\
\hline 5 & Valía personal & .435 & & & & & & .345 \\
\hline 22 & Cólera & & .640 & & & & & .465 \\
\hline 16 & Irritabilidad & & .605 & & & & & .415 \\
\hline 28 & Aburrimiento & & .588 & & & & & .406 \\
\hline 7 & Tristeza & & .586 & & & & & .551 \\
\hline 26 & Preocupado & & .572 & & & & & .462 \\
\hline 17 & Pesimismo & & .512 & & & & & .389 \\
\hline 18 & Fatiga & & .413 & & & & & .383 \\
\hline 25 & Anhedonia general & & & .761 & & & & .646 \\
\hline 10 & Anhedonia pares & & & .759 & & & & .627 \\
\hline 23 & Reducción de la comunice & ición & & .589 & & & & .411 \\
\hline 19 & Autorreproche & & & & .652 & & & .500 \\
\hline 15 & Autoestima & & & & .512 & & & .333 \\
\hline 21 & Compasión de sí mismo & .453 & & & .466 & & & .462 \\
\hline 6 & Aislamiento & & & & .448 & & & .299 \\
\hline 24 & Alteraciones del sueño & & & & & .612 & & .434 \\
\hline 11 & Queja somática & & & & & .544 & & .425 \\
\hline 17 & Queja somática & & & & & .492 & & .445 \\
\hline 29 & Trastornos del apetito & & & & & .409 & & .443 \\
\hline 2 & Ansiedad escolar & & & & & & .769 & .605 \\
\hline \multicolumn{2}{|c|}{ Eigenvalue } & 4.013 & 3.033 & 1.983 & 1.903 & 1.561 & 1.243 & \\
\hline \multicolumn{2}{|c|}{$\%$ Varianza explicada } & 13.377 & 10.111 & 6.609 & 6.345 & 5.212 & 4.142 & \\
\hline \multicolumn{9}{|c|}{ \% Varianza total explicada 45.786} \\
\hline
\end{tabular}

Nota: Sólo se han incluido los pesos factoriales mayores que .40 
donia; el cuarto factor está vinculado a la autoestima, crítica desvalorizada de sí mismo y aislamiento; el quinto factor involucra un componente externalizado somático-vegetativo de la depresión expresado por alteraciones del sueño, del apetito y queja somática y el sexto factor vinculado a la ansiedad provocada por factores escolares. Para comprobar la viabilidad del análisis factorial por sexo y gestión, y poder examinar la validez de constructo de la EDAR, también se empleó la medida de Kaiser-Meyer y Olkin y el test de esfericidad de Barlett, los que arrojaron puntajes y valores significativos que permitieron proseguir con el estudio.

Con la finalidad de examinar las diferencias en la estructura factorial de la EDAR según el sexo, se llevó a cabo un análisis de componentes principales para los ítems de la EDAR (tabla $\mathrm{N}^{\circ}$ 14). Se encontró cargas factoriales similares para el primer factor, tanto para varones como para las mujeres, lo que revela una alta correspondencia entre las cargas factoriales de los ítems de la EDAR. Se aprecia en los varones un eigenvalue de 6.076 para el primer factor, cuya varianza explicada es de 20.254 y para las mujeres el eigenvalue correspondiente fue de 7.767, con una varianza explicada de 25.889 .

Cuando se procedió a la rotación ortogonal de los ítems de la EDAR mediante el método Varimax, en el grupo de varones se llegó a una solución de siete factores cuyos eigenvalues son 2.840,
$2.817,2.162,1.854,1.653,1.539 \mathrm{y}$ 1.126 , respectivamente, siendo la varianza total explicada de 46.649 (tabla $\mathrm{N}^{\mathrm{o}} 16$ ).

El factor 1 se vincula con las expresiones emocionales de cólera, irritabilidad, tristeza, aburrimiento, preocupación, pesimismo y desesperanza; el segundo se asocia a un pobre autoconcepto, sentimientos de autorreproche y autocrítica negativa; el tercero está vinculado a una desvalorización de sí mismo, sentirse no apreciado y con sentimientos de soledad; el cuarto consiste en ítems que sugieren anhedonia; el quinto se relaciona con quejas somáticas; el sexto con aislamiento y alteraciones del sueño y el sétimo factor se asocia a la ansiedad escolar.

En la tabla $\mathrm{N}^{\circ} 17$ se presentan los pesos factoriales rotados de los reactivos de la EDAR (método Varimax), para la submuestra de mujeres se llegó a una solución de seis factores cuyos eigenvalues son 4.689, 3.234, 2.103, 2.101, 1.503 y 1.197 respectivamente, siendo la varianza total explicada de 49.129. El primer factor se encuentra vinculado a sentimientos de desmoralización, desesperanza, compasión de sí mismo y soledad; el segundo es similar al de la muestra total, caracterizándose por expresiones emocionales de tristeza, pesimismo, cólera, preocupación, aburrimiento y fatiga; el tercero se asocia al autorreproche, aislamiento y autoestima; el cuarto sugiere anhedonia; el quinto está vinculado a trastornos somático-vegetativos y 
Tabla № 16

Pesos factoriales rotados de los ítems de la EDAR mediante el método Varimax para la submuestra de varones $(\mathrm{N}=1075)$

\begin{tabular}{|c|c|c|c|c|c|c|c|c|c|}
\hline No. & Ítems & 1 & 2 & 3 & 4 & 5 & 6 & 7 & $h^{2}$ \\
\hline 22 & Cólera & .657 & & & & & & & .491 \\
\hline 16 & Irritabilidad & .609 & & & & & & & .442 \\
\hline 28 & Aburrimiento & .566 & & & & & & & .411 \\
\hline 26 & Preocupado & .551 & & & & & & & .473 \\
\hline 7 & Tristeza & .549 & & & & & & & .488 \\
\hline 17 & Pesimismo & .513 & & & & & & & .420 \\
\hline 18 & Fatiga & & & & & .418 & & & .348 \\
\hline 1 & Disforia & & .435 & & & .435 & & & .526 \\
\hline 8 & Llanto & & .465 & & & & & & .408 \\
\hline 14 & Daño a sí mismo & & .670 & & & & & & .472 \\
\hline 13 & Abatimiento & & .620 & & & & & & .472 \\
\hline 20 & Desaprobarse a sí mismo & & .564 & & & & & & .582 \\
\hline 21 & Compasión de sí mismo & & .464 & & & & & & .455 \\
\hline 4 & Devaluado por padres & & .422 & .457 & & & & & .429 \\
\hline 19 & Autorreproche & & .418 & & & & & & .441 \\
\hline 30 & Desesperanza & & .414 & & & & & & .376 \\
\hline 9 & No apreciado & & .413 & .500 & & & & & .485 \\
\hline 5 & Valía personal & & & .650 & & & & & .492 \\
\hline 12 & Bajo mérito personal & & & .607 & & & & & .516 \\
\hline 3 & Soledad & & & .391 & & & & & .334 \\
\hline 10 & Anhedonia pares & & & & .732 & & & & .550 \\
\hline 25 & Anhedonia general & & & & .717 & & & & .553 \\
\hline 23 & Reducción de la comunic & ación & & & .630 & & & .428 & \\
\hline 29 & Trastornos del apetito & & & & .361 & & & & .482 \\
\hline 27 & Queja somática & & & & & .709 & & & .555 \\
\hline 11 & Queja somática & & & & & .685 & & & .510 \\
\hline 6 & Aislamiento & & & & & & .607 & & .496 \\
\hline 24 & Alteraciones del sueño & & & & & & .471 & & .334 \\
\hline 15 & Autoestima & & & & & & .431 & & .325 \\
\hline 2 & Ansiedad escolar & & & & & & & .824 & .700 \\
\hline \multicolumn{2}{|c|}{ Eigenvalue } & 2.840 & 2.817 & 2.162 & 1.854 & 1.653 & 1.539 & 1.126 & \\
\hline \multicolumn{2}{|c|}{$\%$ Varianza explicada } & 9.467 & 9.392 & 7.026 & 6.182 & 5.118 & 5.131 & 3.754 & \\
\hline \multicolumn{2}{|c|}{$\%$ Varianza total explicada } & 46.649 & & & & & & & \\
\hline
\end{tabular}

Nota: Sólo se han incluido los pesos factoriales mayores que .36. 
Tabla № 17

Pesos factoriales rotados de los ítems de la EDAR mediante el método Varimax para la submuestra de mujeres $(\mathrm{N}=888)$

\begin{tabular}{|c|c|c|c|c|c|c|c|c|}
\hline No. & Items & 1 & 2 & 3 & 4 & 5 & 6 & $h^{2}$ \\
\hline 12 & Bajo mérito personal & 711 & & & & & & .579 \\
\hline 20 & Desaprobarse a sí mismo & 674 & & & & & & .597 \\
\hline 9 & No apreciado & 665 & & & & & & .575 \\
\hline 30 & Desesperanza & 597 & & & & & & .534 \\
\hline 4 & Devaluado por padres & 596 & & & & & & .444 \\
\hline 1 & Disforia & .594 & & & & & & .531 \\
\hline 3 & Soledad & 536 & & & & & & .481 \\
\hline 5 & Valía personal & 524 & & & & & & .437 \\
\hline 21 & Compasión de sí mismo & .519 & & .409 & & & & .492 \\
\hline 14 & Daño a sí mismo & .505 & & & & & & .479 \\
\hline 13 & Abatimiento & 494 & & & & & & .491 \\
\hline 7 & Tristeza & .470 & .545 & & & & & .567 \\
\hline 8 & Llanto & 467 & .432 & & & & & .554 \\
\hline 17 & Pesimismo & & .472 & & & & & .406 \\
\hline 26 & Preocupado & & .612 & & & & & .462 \\
\hline 22 & Cólera & & .610 & & & & & .453 \\
\hline 28 & Aburrimiento & & .595 & & & & & .411 \\
\hline 16 & Irritabilidad & & .585 & & & & & .388 \\
\hline 18 & Fatiga & & .554 & & & & & .418 \\
\hline 19 & Autorreproche & & & .640 & & & & .483 \\
\hline 15 & Autoestima & & & .578 & & & & .406 \\
\hline 6 & Aislamiento & & & .493 & & & & .339 \\
\hline 27 & Queja somática & & & .370 & & 358 & & .382 \\
\hline 11 & Queja somática & & & .358 & & 355 & & .376 \\
\hline 25 & Anhedonia general & & & & 813 & & & .702 \\
\hline 10 & Anhedonia pares & & & & .779 & & & .646 \\
\hline 23 & Reducción de la comunicación & & & & .578 & & & .465 \\
\hline 29 & Trastornos del apetito & & & & & .664 & & .534 \\
\hline 24 & Alteraciones del sueño & & & & & .570 & & .435 \\
\hline 2 & Ansiedad escolar & & & & & & 806 & 672 \\
\hline \multirow{3}{*}{\multicolumn{2}{|c|}{$\begin{array}{l}\text { Eigenvalue } \\
\% \text { Varianza explicada } \\
\% \text { Varianza total explicada }\end{array}$}} & 4.689 & 3.234 & 2.103 & 2.001 & 1.503 & 1.197 & \\
\hline & & 15.630 & 10.781 & 7.044 & 6.671 & 5.011 & 3.991 & \\
\hline & & 49.129 & & & & & & \\
\hline
\end{tabular}

Nota: Sólo se han incluido los pesos factoriales mayores que .35

el sexto factor involucra ansiedad escolar. Los ítems 7 y 8 son compartidos por el primer y segundo factores, el 21 por el primer y tercer factores y el 11 y 27 por el tercer y quinto factores.

Los pesos factoriales no rotados del primer factor, mediante el análisis de componentes principales de los reactivos de la EDAR para las submuestras por tipo de gestión escolar, aparecen en la tabla $\mathrm{N}^{\circ} 18$. Se puede apreciar cargas factoriales similares para los diferentes ítems tanto en los colegios estatales como en los particulares, habiéndose incluido aquellos pesos factoriales superiores a .30 , por lo que se omitieron los items $2,10,23,25$ y 29 , cuyas cargas factoriales eran más bajas. 
Tabla № 18

Pesos factoriales no rotados del primer factor obtenidos en el análisis de los componentes principales de los ítems de la EDAR en la muestra total de estandarización y submuestras por gestión

\begin{tabular}{|c|c|c|c|c|}
\hline $\mathrm{N}^{\circ}$ & İtems & $\begin{array}{c}\text { Muestra total } \\
N=1963\end{array}$ & $\begin{array}{c}\text { Estatal } \\
N=1049\end{array}$ & $\begin{array}{c}\text { Particular } \\
\mathrm{N}=914\end{array}$ \\
\hline 1 & Disforia & 672 & .530 & .604 \\
\hline 2 & Ansiedad escolar & 668 & & \\
\hline 3 & Soledad & 645 & .538 & .593 \\
\hline 4 & Devaluado por padres & 602 & .443 & .479 \\
\hline 5 & Valia personal & .598 & & .447 \\
\hline 6 & Aislamiento & 598 & 408 & .515 \\
\hline 7 & Tristeza & 581 & 615 & 660 \\
\hline 8 & Llanto & 576 & .586 & .586 \\
\hline 9 & No apreciado & 569 & 643 & .666 \\
\hline 10 & Anhedonia pares & 569 & & \\
\hline 11 & Queja somática & 550 & .424 & .380 \\
\hline 12 & Bajo mérito personal & .528 & .547 & .535 \\
\hline 13 & Abatimiento & .506 & 587 & .557 \\
\hline 14 & Daño a sí mismo & 504 & .541 & .489 \\
\hline 15 & Autoestima & 482 & 335 & .514 \\
\hline 16 & Irritabilidad & .475 & .443 & .531 \\
\hline 17 & Pesimismo & .453 & .514 & .587 \\
\hline 18 & Fatiga & 448 & 368 & .463 \\
\hline 19 & Autorreproche & .443 & 364 & .530 \\
\hline 20 & Desaprobarse a sí mismo & 428 & 635 & .692 \\
\hline 21 & Compasión de sí mismo & .425 & .524 & .650 \\
\hline 22 & Cólera & .404 & .387 & .444 \\
\hline 23 & Reducción de la comunicación & 394 & & \\
\hline 24 & Alteraciones del sueño & 385 & .378 & \\
\hline 25 & Anhedonia general & & & \\
\hline 26 & Preocupado & & .499 & .537 \\
\hline 27 & Queja somática & & 419 & .423 \\
\hline 28 & Aburrimiento & & .496 & .498 \\
\hline 29 & Trastornos del apetito & & & \\
\hline 30 & Desesperanza & 337 & 536 & 617 \\
\hline \multicolumn{2}{|c|}{ Eigenvalue } & 6.989 & 6.165 & 7.436 \\
\hline$\% \mathrm{~V}$ & arianza total & 23.296 & 20.549 & 24.786 \\
\hline
\end{tabular}

Nota: Sólo se han incluido los pesos factoriales mayores que 0.30 .

Para el estatal el eigenvalue es de 6.165 , con un porcentaje de varianza total de 20.549 y para el particular el eigenvalue es de 7.436 , con un porcentaje de varianza total de 24.786 .

Los pesos factoriales rotados mediante el procedimiento Varimax, según el tipo de gestión, se presentan en las tablas $\mathrm{N}^{\circ} 19$ y $\mathrm{N}^{\circ} 20$. En la submuestra de la gestión estatal se llegó a una solución de siete factores cuyos eigenva- lues son 3.715, 2.816, 1.695, 1.674, $1.591,1.331$ y 1.252 respectivamente, siendo la varianza total explicada de 46.911, mientras que para la particular se obtuvo seis factores cuyos eigenvalues son: 3.143, 3.136, 2.992, 2.130, 1.589 y 1.274 , siendo la varianza total explicada de 44.204.

Para la gestión estatal (tabla $\left.N^{\circ} 19\right)$ el primer factor se relaciona con sentimientos de desmoralización y desespe- 
Tabla № 19

Pesos factoriales rotados de los ítems de la EDAR mediante el método Varimax para la submuestra de gestión estatal $(\mathrm{N}=1049)$

\begin{tabular}{|c|c|c|c|c|c|c|c|c|c|}
\hline $\mathrm{N}^{0}$ & İtems & F1 & F 2 & F 3 & F 4 & F5 & F 6 & F 7 & $\mathrm{~h}^{2}$ \\
\hline 20 & Desaprobarse a sí mismo & 686 & & & & & & & .552 \\
\hline 12 & Bajo mérito personal & 641 & & & & & & & .512 \\
\hline 9 & No apreciado & 609 & & & & & & & .490 \\
\hline 4 & Devaluado por padres & .564 & & & & & & & .373 \\
\hline 14 & Daño a sí mismo & .546 & & & & & & & .428 \\
\hline 13 & Abatimiento & .543 & & & & & & & .544 \\
\hline 30 & Desesperanza & .491 & & & & & & & .408 \\
\hline 1 & Disforia & .472 & .374 & & & & & & .475 \\
\hline 5 & Valía personal & .455 & & & & & & & .415 \\
\hline 21 & Compasión de sí mismo & .423 & & & & 374 & & & .363 \\
\hline 3 & Soledad & .391 & .359 & & & & & & .359 \\
\hline 8 & Llanto & .382 & 399 & & & & & & .447 \\
\hline 7 & Tristeza & 374 & .561 & & & & & & .524 \\
\hline 22 & Cólera & & .633 & & & & & & .477 \\
\hline 26 & Preocupado & & .596 & & & & & & .492 \\
\hline 16 & Irritabilidad & & .566 & & & & & & .363 \\
\hline 17 & Pesimismo & & .524 & & & & & & .412 \\
\hline 28 & Aburrimiento & & .519 & & & & & & .415 \\
\hline 25 & Anhedonia general & & & .766 & & & & & .619 \\
\hline 10 & Anhedonia pares & & & .755 & & & & & .592 \\
\hline 23 & Reducción de la comunicación & & & & & & .552 & & .482 \\
\hline 27 & Queja somática & & & & 656 & & & & .522 \\
\hline 11 & Queja somática & & & & 609 & & & & .452 \\
\hline 18 & Fatiga & & & & .556 & & & & .415 \\
\hline 19 & Autorreproche & & & & & .587 & & & .504 \\
\hline 6 & Aislamiento & & & & & .571 & & & .460 \\
\hline 15 & Autoestima & & & & & .434 & & & .416 \\
\hline 24 & Alteraciones del sueño & & & & & & .485 & & .440 \\
\hline 29 & Trastornos del apetito & & & & & & 638 & & .487 \\
\hline 2 & Ansiedad escolar & & & & & & & .789 & .635 \\
\hline \multicolumn{2}{|c|}{ Eigenvalue } & 3.715 & 2.816 & 1.695 & 1.674 & 1.591 & 1.331 & 1.252 & \\
\hline \multirow{2}{*}{\multicolumn{2}{|c|}{$\begin{array}{l}\text { \% Varianza explicada } \\
\% \text { Varianza total explicada }\end{array}$}} & 12.383 & 9.386 & 5.650 & 5.581 & 5.302 & 4.437 & 4.173 & \\
\hline & & 46.911 & & & & & & & \\
\hline
\end{tabular}

Nota: Sólo se han incluido los pesos factoriales mayores que .35

ranza; el segundo con expresiones emocionales de cólera, tristeza, pesimismo y aburrimiento; el tercero sugiere anhedonia; el cuarto se asocia con queja somática y fatiga; el quinto se relaciona con autorreproche y aislamiento; el sexto consiste en alteraciones del sueño y trastornos del apetito, y el sétimo factor comprende ansiedad escolar.
Las expresiones de soledad, llanto y tristeza se ubican por sus pesos factoriales, tanto en el factor 1 como en el factor 2 .

Para la gestión particular (tabla $\mathrm{N}^{\circ}$ 20) el primer factor está vinculado a las expresiones emocionales de irritabilidad, tristeza, cólera, pesimismo, aburrimiento; el segundo se asocia a senti- 
Tabla № 20

Pesos factoriales rotados de los ítems de la EDAR mediante el método Varimax para la submuestra de gestión particular $(\mathrm{N}=914)$

\begin{tabular}{|c|c|c|c|c|c|c|c|c|}
\hline $\mathrm{N}^{0}$ & İtems & F 1 & F 2 & F 3 & F 4 & F 5 & F 6 & $h^{2}$ \\
\hline 16 & Irritabilidad & 653 & & & & & & .477 \\
\hline 7 & Tristeza & 628 & .392 & & & & & 5.85 \\
\hline 22 & Cólera & 623 & & & & & & .430 \\
\hline 28 & Aburrimiento & 604 & & & & & & .407 \\
\hline 17 & Pesimismo & .514 & & & & & & .417 \\
\hline 26 & Preocupado & .506 & & & & & & .424 \\
\hline 18 & Fatiga & .494 & & & & & & .452 \\
\hline 8 & Llanto & .466 & .413 & & & & .422 & .590 \\
\hline 3 & Soledad & .418 & .479 & & & & & .446 \\
\hline 1 & Disforia & .384 & .443 & & & & & .487 \\
\hline 9 & No apreciado & & .667 & & & & & .589 \\
\hline 4 & Devaluado por padres & & .634 & & & & & .496 \\
\hline 12 & Bajo mérito personal & & .572 & & & & & .484 \\
\hline 20 & Desaprobarse a sí mismo & & .533 & .564 & & & & .633 \\
\hline 5 & Valía personal & & .440 & & & & & .402 \\
\hline 30 & Desesperanza & & .430 & .381 & & & & .453 \\
\hline 21 & Compasión de sí mismo & & & 659 & & & & .567 \\
\hline 6 & Aislamiento & & & 637 & & & & .470 \\
\hline 19 & Autorreproche & & & 610 & & & & .438 \\
\hline 14 & Daño a sí mismo & & & .545 & & & & .464 \\
\hline 15 & Autoestima & & & .494 & & & & .335 \\
\hline 13 & Abatimiento & & & .431 & & & & .387 \\
\hline 25 & Anhedonia general & & & & .793 & & & .654 \\
\hline 10 & Anhedonia pares & & & & .772 & & & .615 \\
\hline 23 & Reducción de la comunicación & & & & 640 & & & .457 \\
\hline 29 & Trastornos del apetito & & & & .432 & & & .345 \\
\hline 11 & Queja somática & & & & & 649 & & .498 \\
\hline 24 & Alteraciones del sueño & & & & & 649 & & .524 \\
\hline 27 & Queja somática & & & & & .514 & & .422 \\
\hline 2 & Ansiedad escolar & & & & & & .759 & .578 \\
\hline \multirow{3}{*}{\multicolumn{2}{|c|}{$\begin{array}{l}\text { Eigenvalue } \\
\% \text { Varianza explicada } \\
\% \text { Varianza total explicada }\end{array}$}} & 3.413 & 3.136 & 2.992 & 2.130 & 1.589 & 1.274 & \\
\hline & & 11.378 & 10.454 & 9.975 & 7.099 & 5.298 & 4.247 & \\
\hline & & 44.204 & & & & & & \\
\hline
\end{tabular}

Nota: Sólo se han incluido los pesos factoriales mayores que .35

mientos de desmoralización; el tercero sugiere sentimientos de autocompasión y autorreproche, baja autoestima y abatimiento; el cuarto se relaciona con anhedonia; el quinto se vincula con la queja somática y alteraciones del sueño y el sexto está relacionado con la ansiedad escolar y el llanto.

La medida de Kaiser-Meyer-Olkin de la adecuación de la muestra y el test de esfericidad de Barlett, para los seis factores de la EDAR (tabla No 21) fueron significativas y permitieron proseguir con el análisis factorial exploratorio de segundo orden.

En la tabla $\mathrm{N}^{\circ} 22$ se observa el resultado de la rotación de los factores a través del método Ortogonal Varimax, el cual permite apreciar claramente la presencia de dos componentes cuyas 
cargas factoriales exceden a $.50 \mathrm{y}$ explican el 66.231 de la varianza total. Para el factor I el eigenvalue es de 2.956, con una varianza explicada de 49.264. En este factor convergen claramente los subcomponentes: F5 (somato-vegetativo, relacionado con alteraciones del sueño, del apetito y físicas); F2 (expresiones emocionales de cólera, irritabilidad, tristeza, aburrimiento y fatiga.); F1 (desmoralización, desesperanza y sentirse no apreciado), y F5 (baja autoestima, desvalorización de sí mismo y aislamiento). Para el factor II, el eigenvalue es de 1.018, con una varianza explicada de 16.967. Este componente es bipolar, pues involucra en el extremo positivo el F6 (ansiedad esco-

Tabla № 21

Medida de Kaiser-Meyer-Olkin de adecuación de la muestra de estudiantes de secundaria de Lima ( $N=1963)$ y test de esfericidad de Barlett para los factores de la EDAR

\begin{tabular}{ccc}
\hline K-M-O & Test de Bartlett & Significación \\
\hline .804 & 4091.874 & .000 \\
\hline
\end{tabular}

Tabla № 22

Pesos factoriales no rotados y rotados de los factores de la EDAR Análisis factorial confirmatorio de segundo orden

\begin{tabular}{lccccc}
\hline & \multicolumn{2}{c}{ No rotados } & \multicolumn{2}{c}{ Rotados } \\
& 1 & 2 & Factor I & Factor II & $\mathrm{h}^{2}$ \\
\hline Factor 1 & .859 & & .855 & & .739 \\
Factor 2 & .852 & & .856 & & .736 \\
Factor 3 & & -.560 & & -.575 & .399 \\
Factor 4 & .804 & & .800 & & .650 \\
Factor 5 & .861 & & .862 & & .743 \\
Factor 6 & & .825 & & .816 & .706 \\
\hline Eigenvalue & 2.961 & 1.013 & 2.956 & 1.018 \\
\% Var. explicada & 49.356 & 16.875 & 49.264 & 16.967 & \\
\% Var. total explicada & 66.231 & & & & \\
\hline
\end{tabular}

Tabla № 23

Validez de construcción de la prueba EDAR, resultados del análisis factorial confirmatorio de segundo orden $(\mathrm{N}=1963)$

\begin{tabular}{cccc}
\hline Datos & Modelo de 2 factores & Modelo saturado & Modelo independiente \\
\hline Parámetros & 18 & 21 & 6 \\
Cmin & 3.52 & 0 & 4097.79 \\
g.l. & 5 & 0 & 15 \\
p & 0.318 & & 0.000 \\
Cmin/g.l. & 1.18 & 0.00 & 273.19 \\
RMR & 0.03 & 1.00 & 4.74 \\
GFI & 0.99 & & 0.54 \\
AGFI & 0.99 & & 0.36 \\
\hline
\end{tabular}


lar) y en el extremo negativo el F3 (anhedonia).

Los resultados presentados en la tabla $\mathrm{N}^{\circ} 23$ permiten observar que el modelo de dos factores propuestos presenta un valor chi-cuadrado que no es significativo, es decir, que los datos observados no se diferencian de forma marcada de los datos que se esperarían obtener si el modelo fuese adecuado.

Por lo tanto, se concluye que el modelo de dos factores funciona adecuadamente en la muestra.

El análisis de los datos fue realizado con el programa AMOS v 4.0 (Arbuckle, 1999, 2000) y de acuerdo con las recomendaciones propuestas por Byrne
(1998) y por Jöreskog \& Sörbom (1998) se evaluaron el modelo propuesto y dos modelos alternativos, el modelo saturado y el modelo independiente, que son utilizados como criterios de contraste.

El análisis de residuales (RMR), que corresponde a los datos observados una vez que se restan los correspondientes al modelo, permite apreciar que los valores son inferiores al criterio de $0.5, \mathrm{y}$, además, que los estadísticos que evalúan la adecuación del modelo propuesto con los datos observados, como es el caso del índice de bondad de ajuste (GFI) y el índice de bondad de ajuste adaptado (AGFI), son eficientes por ser mayores que 0.90 (Mueller, 1998;

Figura № 2

Análisis factorial confirmatorio de segundo orden

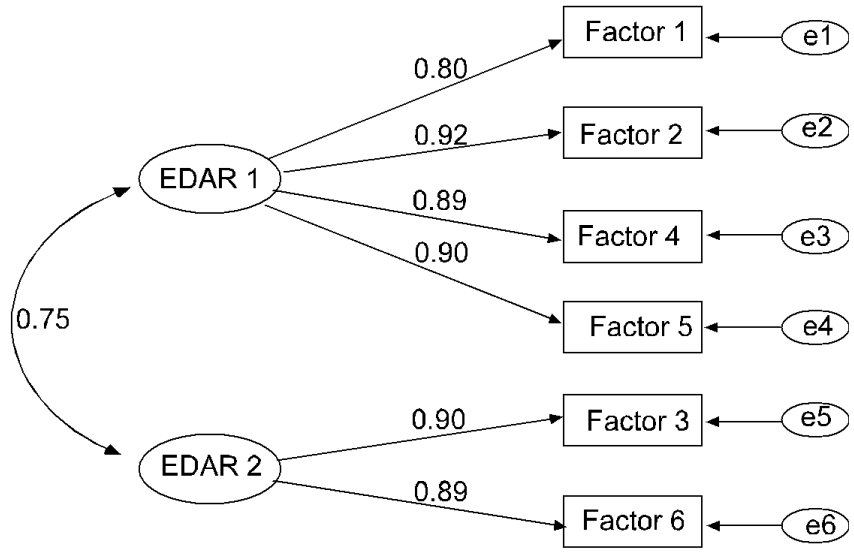

Chi-cuadrado $=3.524$

Grados de libertad $=5$

Nivel de significación $=0.318$ 
Byrne, 1998), por lo que se concluye que el modelo de los dos factores se corrobora en los datos evaluados, lo cual indica que en nuestro medio es válida la estructura factorial de segundo orden de la prueba EDAR.

\section{Normas de interpretación}

En este informe sólo se presentan normas percentilares (baremos) para la muestra total de estandarización (tabla $\mathrm{N}^{\mathrm{o}} 24$ del anexo).

\section{Error estándar de medición}

El error estándar de medición (EEM), obtenido para varias sub-muestras de adolescentes señalan que existe muy poca variabilidad en los resultados, pues la mayoría de los EEM se aproximan a un puntaje bruto de 4 . Para el cálculo se utilizó el coeficiente alfa de Cronbach.

\section{Discusión}

Una multitud de problemas de naturaleza similar a las que manifiestan los adultos depresivos, son asociados con la depresión en adolescentes. Los individuos con depresión tienen dificultad para concentrarse en el trabajo y no logran desarrollar niveles óptimos de competencia. En los adolescentes, la depresión interfiere en el aprendizaje escolar y en su participación en actividades extracurriculares. En los casos más severos, puede traer consigo la desesperanza, ideas suicidas y comportamientos auto- destructivos. Por eso, es importante la identificación temprana del adolescente deprimido, antes de que desarrolle una psicopatología más severa.

Un área muy relacionada con los problemas del comportamiento es el abuso de sustancias psicoactivas. En los adultos es normal encontrar que la depresión puede llevarlos al consumo de alcohol o de otras sustancias para olvidar sus problemas o para aliviar el estrés y psra sentirse mejor. Muchos adolescentes no relacionan su malestar psicológico con la depresión y recurren al alcohol y las drogas como medio de afronte inadecuado para esclarecer su problema. Ellos ignoran que existen programas de tratamiento que brindan una información profunda sobre los desórdenes psicológicos-afectivos que puedan presentárseles.

La depresión es un fenómeno real en los adolescentes y una forma de psicopatología que no se alivia sin intervención. Los programas para la identificación de los adolescentes con un sentimiento de angustia deben ser implementados para disminuir la tasa de estudiantes que se encuentran desalentados en el colegio, la sociedad y en su propia vida. Los procedimientos de identificación deben estar asociados a los problemas de salud mental y ser llevados a cabo a través de los programas de intervención en la escuela. Es evidente que existe una gran necesidad de identificar y brindar un tratamiento conveniente a los adolescentes deprimidos. 
Si los colegios, padres y profesionales están interesados en mantener el bienestar de los adolescentes, deben dirigir más la atención a los estados afectivos de estos jóvenes. En particular, la depresión y la ideación suicida pueden conducir a un comportamiento autodestructivo muy severo que debe ser examinado. La EDAR es un instrumento cuya confiabilidad y validez ha sido probada y que ofrece a los profesionales una evaluación objetiva de la sintomatología depresiva en los adolescentes (Reynolds, 1987).

Los datos que apoyan las características descriptivas, la confiabilidad y la validez de la escala de depresión para adolescentes se presentan en este trabajo y también en el manual profesional de Reynolds, brindando una sólida fundamentación para utilizar este instrumento en el campo de la investigación clínica y educativa.

Está demostrado que la EDAR es una medida confiable, en términos de consistencia interna y de estabilidad. Así, los coeficientes alfa para el puntaje total cuando el ítem de la EDAR es eliminado en la muestra total y submuestras, denotan una alta consistencia interna de la escala en la medición de una muestra relativamente homogénea del dominio general de la depresión, apreciándose, además, correlaciones por encima de .84 para los 30 reactivos de la escala.

El coeficiente de consistencia interna, obtenido por otros investigadores independientes, en distintas áreas geográfi- cas de Estados Unidos, apoyan aún más la alta confiabilidad de la EDAR, que se encuentra entre .90 y .95 .

La confiabilidad por el método de mitades, para la muestra total de estandarización, fue de .85 corregido por la fórmula de Spearman-Brown, lo que es también un indicador de la consistencia de las respuestas a los reactivos que evalúan la sintomatología depresiva de la escala.

La confiabilidad test-retest, después de un periodo de intervalo corto de dos semanas, presenta una correlación elevada (.89), mostrando que la EDAR es una medida estable de la sintomatología depresiva, a pesar de la naturaleza compleja y fluctuante del constructo que se está midiendo. Sin embargo, estos resultados se deben tomar con cautela porque los síntomas de la depresión, particularmente los estados de ánimo pueden demostrar un nivel moderado de cambio por la acción de factores externos, como situaciones específicas de interacción a través del tiempo o algún tipo de enfermedad.

Para la determinación de la validez, se ha recurrido a diferentes procedimientos. En primer lugar, el examen lógico de la escala permite visualizar la correspondencia entre el contenido de los ítems y la sintomatología específica que evalúa. Sin embargo cabe destacar que si bien un síntoma puede ser característico de la depresión, si una gran proporción de adolescentes lo presenta, no debe necesariamente significar dis- 
criminación entre las respuestas de adolescentes depresivos y normales, sino que el ítem puede estar señalando un componente normal del estatus afectivo de los adolescentes. Además, cabe recordar que esta prueba no se basa en una teoría específica de la depresión y no todos los síntomas asociados a ella son evaluados por la prueba.

Por otro lado, cuando se interpreta el coeficiente de correlación ítem-escala total, es conveniente tener en cuenta que la magnitud de la correlación es atenuada por la limitada distribución de las respuestas del ítem, que reduce la variabilidad del puntaje. Típicamente las correlaciones ítem-total en el rango de .30 a .50 son consideradas valiosas; pudiéndose afirmar que existe una buena evidencia que sustenta la validez de contenido de la EDAR como una medida de la depresión en los adolescentes.

La validez concurrente fue el procedimiento más apropiado que se utilizó para la validación de la escala en Estados Unidos, siendo el propósito principal evaluar la sintomatología depresiva del adolescente común, con miras a predecir cuán depresivo podría estar en algún momento futuro. Un criterio empleado en la validación concurrente fue la utilización de la escala de depresión de Hamilton como criterio de medida. Esta escala de clasificación se aplicó a 111 adolescentes de colegios secundarios urbanos y no urbanos, los sujetos fueron seleccionados incluyendo a es- tudiantes depresivos y normales; la edad promedio de la muestra era de 15.5 años (D.S. = 0.67). Cinco entrevistadores experimentados administraron la escala de clasificación de Hamilton y la EDAR para evaluar a los estudiantes. La correlación entre los puntajes de ambas escalas fue .83 (p.<.001). Una réplica en los mismos sujetos a los cuales se aplicó el test en la primera fase del estudio con otros entrevistadores que administraron el Hamilton, obtuvieron similares resultados que dieron mayor soporte a la validez concurrente de la prueba.

Para estudiar la validez de constructo de la EDAR, Reynolds presenta en el manual profesional los resultados de una serie de investigaciones sobre la validez convergente y discriminante, validez factorial, eficacia clínica y sensibilidad a la manipulación del tratamiento. Los estudios están basados en más de 11.000 adolescentes de distintas áreas geográficas, así como de grupos étnicos y socioeconómicos distintos.

Los hallazgos demuestran validez convergente con otras medidas de autorreporte, como la Escala de Depresión de Beck (DBI); la Escala para Estudios Epidemiológicos sobre la Depresión (CES-D); la Escala de Zung y el Inventario de Depresión para Niños de Kovacs (CDI). Las correlaciones oscilaban entre .68 a .76 ( $\mathrm{p}<.0001)$.

La validez convergente también señala relaciones que van de moderadas a intensas entre la EDAR y variables que 
representan correlatos cognitivo-afectivos de la depresión, tales como la autoestima y ansiedad, desesperanza aprendida, soledad e ideas suicidas.

La validez discriminante ha revelado baja magnitud entre la EDAR y mediciones de variables que teóricamente no se relacionan con el autorreporte de la depresión, como la deseabilidad social y el rendimiento académico.

La depresión es un desorden psicológico serio, experimentado por miles de adolescentes, la evidencia de los datos sugiere que la mayoría de estos jóvenes no tienen conciencia del riesgo de desarrollar una patología seria. La determinación de la validez de constructo ha sido desarrollada a través de múltiples investigaciones que proveen evidencia de la validez convergente, demostrada en las relaciones de la EDAR y otros cuatro autorreportes de las medidas de depresión. Otras evidencias para la validez convergente se han indicado sobre la base de fuertes relaciones encontradas entre la EDAR y medidas relacionadas con el constructo, incluyendo: autoestima, ansiedad, ideaciones suicidas, soledad, desesperanza aprendida, así como también medidas de eventos vitales y sociales de mayor o menor magnitud. La evidencia de una validez de discriminación se ha demostrado a través de una relación pobre entre la escala de depresión de adolescentes y las variables de deseabilidad social.

La validez clínica, ha sido sustentada sobre la base del diagnóstico eficaz del puntaje de corte de la EDAR, cuando éste es comparado con el nivel clínico de la depresión establecido en una entrevista clínica, así como también por la sensibilidad de las respuestas de la EDAR para experimentar variaciones por efectos del tratamiento para la depresión.

Otras evidencias para la validez de la escala se muestran a través de la interpretación de los factores de la estructura de los ítems, en la muestra de estandarización peruana. El análisis exploratorio de factores, en el primer componente no rotado para la muestra total y submuestras por sexo y gestión escolar, revela una alta correspondencia entre los ítems de la escala acorde con lo que se esperaba para una medida homogénea de la depresión, como la EDAR.

Cuando se recurre a la rotación ortogonal mediante el método Varimax, si bien se llega a diferentes soluciones en las submuestras, desde un punto de vista cualitativo, se mantiene en gran medida la misma explicación con relación a los factores hallados.

El análisis exploratorio de segundo orden con respecto a la solución de los seis factores inicialmente obtenidos, pero rotados por medio del procedimiento ortogonal Varimax, sugiere la existencia de dos componentes, cuyas cargas factoriales exceden de .50 . y que explican el 66.231 de la varianza total. El primer componente establece una relación de dependencia entre los factores: F1 (desmoralización y desespe- 
ranza); F2 (expresiones emocionales de cólera, tristeza y desánimo); F4 (baja autoestima y desvalorización de sí mismo), y F5 (somático-vegetativo). El segundo componente es bipolar y reúne en un extremo a F3 (anhedonia) y en el otro a F6 (ansiedad escolar).

El análisis factorial confirmatorio de segundo orden, realizado a través del programa Amos versión 4.0, permitió corroborar la adecuación de los dos factores anteriormente identificados, lo cual permite concluir que la EDAR es un instrumento válido desde el punto de vista teórico.

En resumen, este trabajo presenta una investigación extensa sobre la sintomatología depresiva de los adolescentes, un análisis psicométrico riguroso y una exploración de las características clínicas de la EDAR. Es la primera escala de depresión para adolescentes, cuya adaptación y estandarización peruana ha sido desarrollada para la medida de la depresión en una muestra representativa de adolescentes escolares de Lima metropolitana. También se ofrece al usuario de la EDAR una información básica y general acerca de la depresión, poniendo énfasis en su naturaleza y evaluación. Es de interés de los autores del presente estudio que este instrumento sea indispensable para medir la depresión en adolescentes y que su uso sea promovido en los servicios de salud mental para jóvenes.

El conocimiento de la depresión en adolescentes se encuentra aún en sus inicios, pero seguramente es uno de los problemas mayores en el área de la salud mental. La estandarización de una medida de evaluación como la EDAR es el primer paso en torno a su estudio sistemático, ya que hay cientos de jóvenes angustiados que necesitan evaluación y tratamiento. En las escuelas la aplicación de esta escala ha sido vista de manera positiva por maestros, considerándose un método efectivo para identificar los niveles de depresión que pueden estar asociados a problemas mentales potenciales, bajo rendimiento académico, problemas de comportamiento, abuso de sustancias psicoactivas o cualquier otra dificultad. De ahí que se considere una herramienta clínica muy valiosa para los psicólogos, consejeros, trabajadores sociales, enfermeras y otros profesionales de la salud mental y del campo educativo. 


\section{REFERENCIAS}

Abou Nazel, M.; Fahmy, S.; Younis, I.; Seif el-Din, A.; Abdel Fatah, M.; Mokhtar, S. \& Ayoub, A. (1991). A study of depression among Alexandria preparatory school adolescents. Journal of the Egyptian Public Health Association, 66, 649-674.

Albert, N. \& Beck, A. (1975). Incidence of depression in early adolescence: A preliminary study. Journal of Youth and Adolescents, 4, 301-307.

American Psychiatric Association. (1980). Diagnostic and statistical manual of mental disorders $\left(3^{\text {rd }}\right.$ ed.). Washington DC: Author.

Arbuckle, J. (1999). AMOS 4.0: Programming reference guide. Chicago: Small Waters Corporation.

Arbuckle, J. (2000). Introduction to structural equation modeling using AMOS. Austin: Academic Corporation and Instructional Technology Services.

Beck, A. (1976). Cognitive therapy and the emotional disorders. New York: International Universities Press.

Beck, A. \& Beamesdesfer, A. (1974). Assesment of depression: The depression inventory. Modern Problems in Pharmacopsychiatry, 7, 5-19.

Beck, A.; Steer, R. \& Garbin, M. (1988). Psychometric properties of the Beck Depression Inventory: Twenty five year of evaluation. Clinical Psychology Review, 8, 77-100.

Beck, A.; Ward, C.; Mendelson, M.; Mock, J. \& Erbaugh, J. (1961). An inventory for measuring depression. Archives of General Psychiatry, 4, 561-571.

Berganza, C. \& Aguilar, G. (1992). Depression in Guatemalan adolescents. Adolescence, 27 (108), 771-781.

Bernal, G.; Bonilla, J. \& Santiago, J. (1995). Confiabilidad interna y validez de construcción lógica de dos instrumentos para medir sintomatología psicológica en una muestra clínica: El Inventario de Depresión de Beck y la Lista de Cotejo de Síntomas - 36. Revista Latinoamericana de Psicología, 27, 207-229.

Brown, Ch. (1980). Principios de la medición en psicología y educación. México: El Manual Moderno.

Butcher (1995). Clinical personality assessment: Practical approaches. New York: Oxford University Press.

Byrne, B.M. (1989). A primer of LISREL: Basic applications and programming for confirmatory analytic models. New York: Springer-Verlag Inc.

Byrne, B.M. (1998). Structural equation modeling with LISREL, PRELIS, and SIMPLIS: Basic concepts, applications and programming. New Jersey: Lawrence Erlbaum Associates Publishers.

Carlson, G. \& Strober, M. (1979). Affective disorders in adolescents. Psychiatric Clinics of North America, 21, 361-368.

Cattell, R. (1966). The scree test for number of factors. Multivariate Behavioral Research, 1, 245-276.

Cervantes, R.; Padilla, A. \& Salgado, N. (1990). Reliability and validity of the Hispanic Stress Inventory. Hispanic Journal of Behavioral Sciencies, 12, 76-82.

Cronbach, L. (1951). Coefficient alpha and the internal structures of tests. Psychometrika, 16, 297-334.

Cytryn, L. \& Mc Knew, D. (1972). Proposed classification of chilhood depression. American Journal of Psychiatric, 120, 149-155.

Christ, A.; Adler, A.; Isacoff, M. \& Gershansky, I. (1981). Depression: Symptoms versus diagnosis in 10,412 hospitalized children and adolescents (1957- 
1977). American Journal of Psychotherapy, 35, 400-412.

DSM IV (1995). Manual de diagnóstico y estadística de las enfermedades psiquiátricas. Barcelona: Masson, S.A.

Endicott, J.; Cohen, J.; Nee, J.; Fleiss, J. \& Saratakos, S. (1981). Hamilton Depression Rating Scale extracted from regular and changed versions of the Schedule for Affective Disorders and Schizophrenia. Archives of General Psychiatry, 38, 98-103.

Endicott, J. \& Spitzer, R. (1978). A diagnostic interview: The schedule for affective disorders and schizophrenia. Archives of General Psychiatry, 35, 837-844.

Friedman, R.; Clarkin, J.; Corn, R.; Aronoff, M.; Hurt, S. \& Murphy, M. (1982). DSM-III and affective pathology in hospitalized adolescents. Journal of Nervous and Mental Disorders, 170, 511-521.

Friedman, R.; Hurt, S.; Clarkin, J.; Corn, R. \& Aronoff, M. (1983). Symptoms of depression among adolescents and young adults. Journal of Affective Disorders, 5, 37-43.

Gallagher, D.; Nies, G., \& Thompson, L. (1982). Reliability of the Beck Depression Inventory with older adults. Journal of Consulting and Clinical Psychology, 50, 152-153.

Glaser, K. (1967). Masked depresión in children and adolescents. American Journal of Psychotherapy, 21, 565574.

Hamilton, M. (1960). A rating scale for depression. Journal of Neurology, Neurosurgery and Psychiatry, 23, 56-62.

Hamilton, M. (1967). Development of a rating scale for primary depressive illness. Bristish Journal of Social and Clinical Psychology, 6, 278-296.
Hollon, T. (1970). Poor school performance as a sympton of masked depression in children and adolescents. American Journal of Psychoterapy, 24, 258-263.

Instituto de Investigaciones Psicológicas (1996). Algunas características psicosociales de la juventud en la Región Metropolitana del Valle Central de Costa Rica. San José: Universidad de Costa Rica.

Jöreskog, K. \& Sörbom, D. (1998). LISREL 8: Structural Equation Modeling with the SIMPLIS Command Language. New Jersey: Lawrence Erlbaum Associates Publishers.

Kashani, J., McGee, R., Clarkson, S., et al. (1983). Depression in a sample of nine year old children. Arch. Gen. Psychiatric, 40, 1217-1223.

Kaplan, S.; Hong, G. \& Weinhold, C. (1984). Epidemiology of depressive symptomatology in adolescents. Journal of the American Academy of Child Psychiatry, 23, 91-98.

Kerlinger, F. (1994). Investigación del comportamiento. México: McGraw Hill.

Kovacs, M. (1989). Affective disorders in children and adolescents. American Psychologist, 44, 209-215.

Kovacs, M. (1979). Children's Depression Inventory. Pittsburg: University of Pittsburg School of Medicine.

Kovacs, M.; Feinberg, T.; Crouse-Novak, M.; Paulauskas, S.; Pollock, M. \& Finkelstein, R. (1984). Depressive disorders in chilhood: II. A longitudinal study of the risk for subsequent mayor depression. Archives of General Psychiatriy, 41, 229-237.

Larsson, B. \& Melin, L. (1992). Prevalence and short-term stability of depressive symptoms in schoolchildren. Acta Psychiatrica Scandinavica, 85, 17-22. 
Lorente, A. (1988). La depresión. Barcelona: Martínez Roca.

Lugo, I.; Louro I. \& Bayarre, H. (1998). La depresión en Cuba. Validación del Inventario de Depresión de Beck de la Escala de Actitudes Disfuncionales en población cubana. Avances en Psicología Clínica Latinoamericana, 111-120.

Myers, J.; Weissman, M.; Tischler, G., et al (1984). Six months prevalence of psychiatric disorders in three communities. Arch. Gen. Psychiatry, 41, 959-967.

Mueller, R.O. (1998). Basic principles of structural equation modeling: An introduction to LISREL and EQS. New York: Springer-Verlag Inc.

Novaco, R. (1975). Anger control: The development and evaluation of an experimental treatment. Lexington: Lexington Books / D.C. Heath.

Novara, J.; Sotillo, C. \& Warthon, D. (1983). Estandarización y correlación de las escalas de Beck, Hamilton y Zung para depresión en población de Lima metropolitana. Lima: Instituto Nacional de Salud Mental Honorio Delgado-Hideyo Noguchi.

Reátegui, L. (1994). La depresión infantil. Estandarización del Inventario de Depresión para Niños (CDI). Tesis para optar el título de licenciada en psicología. Universidad Peruana Cayetano Heredia.

Rehm, L. (1981). Assesment depression. En: M. Hersen \& A. Bellack (Eds.). Behavioral assessment: A practical handbook. $2^{\text {nd }}$ ed., 246-295. New York: Pergamon Press.

Reynolds, W. (1983). Depression in adolescents: Measurement, epidemiology and correlates. Paper presented at the annual meeting of the National Association of School Pychologists, Detroit.

Reynolds, W. (1985). Depression in childhood and adolescence: Diagnosis, as- sessment, intervention strategies and reasearch. En: T.R. Kratochwill (Ed.). Advances in school psychology, Vol. 4, 133-189. Hillsdale, NJ: Lawrence Eribaum.

Reynolds, W. (1986a). A model for the screening and identification of depressed children and adolescents in school settings. Professional School Psychology, 1, 117-129.

Reynolds, W. (1987). Reynolds Adolescent Depression Scale. Professional Manual. Psychological Assessment Resources, Inc. PAR.

Reynolds, W. \& Bartell, N. (1984). Stability of depressive symtomatology in adolescents. Unpublished manuscript.

Reynolds, W. \& Coats, K. (1986). A comparison of cognitive-behavioral therapy and relaxation training for the treatment of depression in adolescents. Journal of Consulting and Clinical Psychology, 49, 306-307.

Reynolds, W., \& Gould, J. (1981). A psychometric investigation of the standard and short form Beck Depression Inventory. Journal of Consulting and Clinical Psychology, 49, 306-307.

Reynolds, W. \& Waltz, J. (1984a). Adolescents Hassles Inventory. Unpublished manuscript.

Rie, H. (1966). Depression in chilhood: A survey of some pertinent contributions. Journal of the American Academy of Child Psychiatry, 21, 251-255.

Robbins, D.; Alessi, N.; Cook, S.; Poznanski, E. \& Yanchyshyn, G. (1982). The use of the Research Diagnostic Criteria (RDC) for depression in adolescent psychiatric inpatients. Journal of the American Acadamy of Child Psychiatry, 21, 251-255.

Robbins, L.; Helzer, J.; Weissman, M.; Orvaschel, H.; Gruenberg, E.; Burke, J. 
\& Regier, D. (1984). Lifetime prevalence of specific psychiatric disorders in three sites. Archives of General Psychiatry, 41, 949-958.

Shaffi, M. \& Shaffi. Depresión en niños y adolescentes: Clínica, evaluación y tratamiento. Madrid: Martínez Roca, S.A.

Spitzer, R.; Endicott, J. \& Robins, E. (1978). Research diagnostic criteria: Rationale and reliability. Archives of General Psychiatry, 35, 773-782.

Strober, M.; Green, J. \& Carlson, G. (1981). Phenomenology and subtypes of mayor depressive disorders in adolescents. Journal of Affective Disorders, 3, 281-290.

Teri, L. (1982). Depression in adolescence: Its relationship to assertion and various aspects of self-image. Journal of Clinical Child Psychology, 11, 101-106.

Ugarriza, N. (1998). Normalización del Inventario de Depresión de Beck (IDB) en estudiantes universitarios de Lima metropolitana. En impresión.

Weinberg, W.; Rutman, J.; Sullivan, L.; Penick, E. \& Dietz, S. (1973). Depression in children referred to an educational diagnostic center: Diagnosis and treatment. Journal of Pedriatrics, 83, 1065-1072.

Whitaker, A.; Johnson, J.; Shaffer, D.; Rapoport, J.; Kalikow, K.; Walsh, B.; Davies, M.; Braiman, S. \& Dolinsky, A. (1990). Uncommon troubles in young people: prevalence estimates of selected Psychiatric Disorders in a nonrefered adolescent population. Archives of General Psychiatric, 47, 487-496.

Yanchyshyn, G. \& Robbins, D. (1983). The assessment of depression in normal adolescents: A comparison study. $\mathrm{Ca}$ nadian Journal of Psychiatry, 28, 522526. 


\section{Anexo}

Tabla № 24

Conversión de los puntajes directos a rangos percentiles para la muestra total y por sexo

\begin{tabular}{|c|c|c|c|c|}
\hline $\begin{array}{l}\text { Puntaje } \\
\text { directo }\end{array}$ & $\begin{array}{c}\text { Total } \\
(\mathrm{N}=1963)\end{array}$ & $\begin{array}{c}\text { Masculino } \\
(\mathrm{N}=1075)\end{array}$ & $\begin{array}{c}\text { Femenino } \\
(\mathrm{N}=888)\end{array}$ & $\begin{array}{l}\text { Puntaje } \\
\text { directo }\end{array}$ \\
\hline 92 & 99 & 99 & 99 & 92 \\
\hline 91 & 99 & 99 & 98 & 91 \\
\hline 90 & 98 & 99 & 98 & 90 \\
\hline 88 & 98 & 99 & 97 & 88 \\
\hline 87 & 98 & 99 & 96 & 87 \\
\hline 86 & 97 & 99 & 95 & 86 \\
\hline 85 & 97 & 98 & 95 & 85 \\
\hline 83 & 97 & 98 & 95 & 83 \\
\hline 82 & 96 & 98 & 93 & 82 \\
\hline 81 & 95 & 98 & 92 & 81 \\
\hline 80 & 94 & 97 & 91 & 80 \\
\hline 79 & 93 & 97 & 89 & 79 \\
\hline 78 & 93 & 96 & 89 & 78 \\
\hline 77 & 92 & 96 & 88 & 77 \\
\hline 76 & 92 & 95 & 87 & 76 \\
\hline 75 & 91 & 95 & 85 & 75 \\
\hline 74 & 90 & 94 & 84 & 74 \\
\hline 73 & 88 & 93 & 82 & 73 \\
\hline 72 & 87 & 93 & 81 & 72 \\
\hline 71 & 86 & 91 & 80 & 71 \\
\hline 70 & 84 & 90 & 77 & 70 \\
\hline 69 & 83 & 89 & 76 & 69 \\
\hline 68 & 81 & 87 & 74 & 68 \\
\hline 67 & 79 & 85 & 72 & 67 \\
\hline 66 & 77 & 83 & 70 & 66 \\
\hline 65 & 75 & 81 & 67 & 65 \\
\hline 64 & 73 & 80 & 65 & 64 \\
\hline 63 & 70 & 77 & 62 & 63 \\
\hline 62 & 67 & 74 & 58 & 62 \\
\hline 61 & 64 & 71 & 56 & 61 \\
\hline 60 & 60 & 67 & 52 & 60 \\
\hline 59 & 57 & 63 & 48 & 59 \\
\hline 58 & 53 & 59 & 45 & 58 \\
\hline 57 & 49 & 57 & 41 & 57 \\
\hline 56 & 45 & 53 & 36 & 56 \\
\hline 55 & 41 & 49 & 33 & 55 \\
\hline 54 & 37 & 43 & 29 & 54 \\
\hline 53 & 33 & 38 & 27 & 53 \\
\hline 52 & 30 & 34 & 24 & 52 \\
\hline
\end{tabular}


Tabla № 24

Conversión de los puntajes directos a rangos percentiles para la muestra total y por sexo

(continuación)

\begin{tabular}{ccccc}
\hline $\begin{array}{c}\text { Puntaje } \\
\text { directo }\end{array}$ & $\begin{array}{c}\text { Total } \\
(\mathrm{N}=1963)\end{array}$ & $\begin{array}{c}\text { Masculino } \\
(\mathrm{N}=1075)\end{array}$ & $\begin{array}{c}\text { Femenino } \\
(\mathrm{N}=888)\end{array}$ & $\begin{array}{c}\text { Puntaje } \\
\text { directo }\end{array}$ \\
\hline 51 & 27 & 31 & 22 & 51 \\
50 & 23 & 27 & 19 & 50 \\
49 & 21 & 23 & 17 & 49 \\
48 & 18 & 20 & 15 & 48 \\
47 & 15 & 18 & 13 & 47 \\
46 & 12 & 14 & 10 & 46 \\
45 & 10 & 11 & 8 & 45 \\
44 & 8 & 9 & 7 & 44 \\
43 & 6 & 7 & 6 & 43 \\
42 & 5 & 5 & 5 & 42 \\
41 & 4 & 4 & 3 & 41 \\
40 & 2 & 1 & 2 & 40 \\
39 & 1 & 1 & 2 & 39 \\
38 & 1 & 57.1860 & 1 & 38 \\
\hline Media & 59.1594 & 10.3055 & 61.5484 & Media \\
D.E. & 11.6147 & 3.9913 & 12.6240 & D.E. \\
E.E.M. & 4.1877 & 4.1869 & E.E.M. \\
\hline
\end{tabular}




\section{Sobre mi mismo \\ por William M. Reynolds}

Nombre:

$\begin{array}{ll}\text { Edad: } & \text { Sexo: } \\ \text { Colegio: Grado:- } & \\ \text { Fecha: } & \end{array}$

\section{Instrucciones}

A continuación se presenta una lista de oraciones sobre cómo te sientes. Lee cada una y decide sinceramente cuán a menudo te sientes así: Casi nunca, Rara vez, Algunas veces o Casi siempre. Marca el círculo que está debajo de la respuesta que mejor describe cómo te sientes realmente. Recuerda, que no hay respuestas correctas ni equivocadas. Sólo escoge la respuesta que dice cómo te sientes generalmente.

\begin{tabular}{|c|c|c|c|c|c|}
\hline & & $\begin{array}{c}\text { Casi } \\
\text { nunca }\end{array}$ & $\begin{array}{c}\text { Rara } \\
\text { vez }\end{array}$ & $\begin{array}{c}\text { Algunas } \\
\text { veces }\end{array}$ & $\begin{array}{c}\text { Casi } \\
\text { siempre }\end{array}$ \\
\hline 1. & Me siento feliz & $\mathrm{O}$ & $\mathrm{O}$ & O & $\mathrm{O}$ \\
\hline 2. & Me preocupa el colegio & O & O & O & O \\
\hline 3. & Me siento solo & O & O & O & O \\
\hline 4. & Siento que mis padres no me quieren & O & O & O & O \\
\hline 5. & Me siento importante & $\mathrm{O}$ & ○ & ○ & O \\
\hline 6. & Siento ganas de esconderme de la gente & O & O & O & O \\
\hline 7. & Me siento triste & O & O & O & O \\
\hline 8. & Me siento con ganas de llorar & 0 & 0 & 0 & 0 \\
\hline 9. & Siento que no le importo a nadie & O & O & O & O \\
\hline 10. & Tengo ganas de divertirme con los compañeros & O & O & 0 & O \\
\hline 11. & Me siento enfermo & O & O & O & O \\
\hline 12. & Me siento querido & O & O & 0 & O \\
\hline 13. & Tengo deseos de huir & $\mathrm{O}$ & O & O & 0 \\
\hline 14. & Tengo ganas de hacerme daño & O & O & O & O \\
\hline 15. & Siento que no les gusto a los compañeros & O & O & O & O \\
\hline 16. & Me siento molesto & O & O & O & O \\
\hline & Siento que la vida es injusta & O & O & O & O \\
\hline 18. & Me siento cansado & O & O & O & O \\
\hline 19. & Siento que soy malo & O & ○ & O & O \\
\hline & Siento que no valgo nada & $\mathrm{O}$ & 0 & O & 0 \\
\hline & Tengo pena de mí mismo & O & O & O & O \\
\hline 22. & Hay cosas que me molestan & O & O & O & O \\
\hline 23. & Siento ganas de hablar con los compañeros & O & O & O & O \\
\hline & Tengo problemas para dormir & 0 & 0 & 0 & 0 \\
\hline & Tengo ganas de divertirme & O & O & O & O \\
\hline & Me siento preocupado & O & O & O & O \\
\hline & Me dan dolores de estómago & 0 & 0 & O & 0 \\
\hline 28. & Me siento aburrido & O & O & O & O \\
\hline & Me gusta comer & O & O & 0 & O \\
\hline 30. & Siento que nada de lo que hago me ayuda & O & O & O & O \\
\hline
\end{tabular}

\begin{tabular}{|c|l|}
\hline RS & \\
\hline TOTAL $\%$ & \\
\hline$\%$ & \\
\hline
\end{tabular}

\begin{tabular}{|l|l|}
\hline \multicolumn{2}{|c|}{$\mathrm{Cl}$} \\
\hline & \\
\hline & \\
\hline
\end{tabular}

